\title{
Radio emission from the A3571 cluster complex: The final stage of a cluster merger?
}

\author{
T. Venturi ${ }^{1}$, S. Bardelli ${ }^{2}$, M. Zagaria ${ }^{1,3}$, I. Prandoni ${ }^{1,3}$, and R. Morganti ${ }^{4}$ \\ 1 Istituto di Radioastronomia, CNR, Via Gobetti 101, 40129 Bologna, Italy \\ 2 Osservatorio Astronomico, Via Ranzani 1, 40126 Bologna, Italy \\ 3 Dipartimento di Astronomia, Università di Bologna, Via Ranzani 1, 40126 Bologna, Italy \\ 4 NFRA, Postbus 2, A7990 Dwingeloo, The Netherlands
}

Received 27 September 2001 / Accepted 15 January 2002

\begin{abstract}
In this paper we report and discuss the results of a radio survey in the A3571 cluster complex, a structure located in the Shapley Concentration core, and formed by the three clusters A3571, A3572 and A3575. The survey was carried out simultaneously at $22 \mathrm{~cm}$ and $13 \mathrm{~cm}$ with the Australia Telescope Compact Array, and led to the detection of 124 radio sources at $22 \mathrm{~cm}$. The radio source counts in this region are in agreement with the background counts. Among the 36 radio sources with optical counterpart, six have a measured redshift that places them at the distance of the A3571 cluster complex, and nine radio sources have optical counterparts most likely members of this cluster complex. All of the radio galaxies emit at low power level, i.e. $\log P_{22 \mathrm{~cm}}\left(\mathrm{~W} \mathrm{~Hz}{ }^{-1}\right)$ $\leq 22.6$. A number of them are likely to be starburst galaxies. The radio luminosity function of early type galaxies is in agreement with that derived by Ledlow \& Owen (1996) if we restrict our analysis to the A3571 cluster. On the basis of the multiwavelength properties of the A3571 cluster complex, we propose that it is a very advanced merger, and explain the radio properties derived from our study in the light of this hypothesis.
\end{abstract}

Key words. radio continuum: galaxies - galaxies: clusters: general - galaxies: clusters: individual: A3571, A3572, A3575

\section{Introduction}

Merging between clusters is thought to be responsible for significant changes in the physics of the intracluster medium and in the emission properties of the galaxy population, as a consequence of the enormous energy budget involved $\left(\sim 10^{60} \mathrm{ergs}\right)$.

The effects of this dynamical process on the hot gas are clear and well documented (Sarazin 2000), as is the connection between cluster merging and peculiar radio sources such as relics and halos (Feretti \& Giovannini 1996). In particular, these extended radio sources are found to be associated with clusters with some degree of disturbance, suggesting that merging is responsible for the re-acceleration of the relativistic electrons (Feretti \& Giovannini 2002; Ensslin \& Brüggen 2002; Brunetti et al. 2001; Buote 2001). On the other hand it is not yet understood whether cluster-cluster collision is able to modify the radio emission properties of a single galaxy and/or affect the statistics of the radio source population.

In order to address this issue, we are carrying on a multiwavelength study of the central part of the Shapley

Send offprint requests to: T. Venturi,

e-mail: tventuri@ira.cnr.it
Concentration, where an unusually high number of clusters are merging. This situation originated from the high local density, that induced peculiar velocities of the order of $\sim 1000 \mathrm{~km} \mathrm{~s}^{-1}$.

Zucca et al. (1993) performed a percolation analysis of the Abell-ACO clusters in order to find superclusters and to study the grouping characteristics as a function of the overdensity with respect to the mean density of clusters. In particular, they found that at the highest density contrasts $(>40)$, the central part of the Shapley Concentration is fragmented in three main structures ("cluster complexes" or "groups of clusters"). One is dominated by the cluster A3558 and could be considered the core of the supercluster; a second is formed by A3528, A3530 and A3532. Both complexes present very clear signs of ongoing merging (Hanami et al. 1999; Bardelli et al. 1996; Ettori et al. 1997; Kull \& Böhringer 1999; Schindler 1996). The third group of clusters in this region is dominated by A3571, and includes A3572 and A3575.

We surveyed the A3558 and A3528 cluster regions at 22 $\mathrm{cm}$ with the Australia Telescope Compact Array (ATCA) to the limiting flux density of $\sim 0.8 \mathrm{mJy}$ (Venturi et al. 2000,2001 ). It was found that the A3558 complex is characterised by a significant lack of radio sources with respect 
to the cluster sample of Ledlow \& Owen (1996), suggesting the possibility that cluster-cluster collisions would "swith off" the existing radio sources, or inhibit the birth of new ones. On the contrary, the number of radio sources found in the A3528 complex is statistically consistent with that one of the control sample. These two different behaviours can be possibly explained in terms of merging age: the A3558 complex is at an advanced stage (possibly after the first core-core encounter, Bardelli et al. 1998), while the A3528 complex is an early merging, where clusters just started to interact (see for example the simulations by Ricker \& Sarazin 2001).

In this paper we present the radio-optical analysis of the third group of clusters in the central part of the Shapley Concentration, i.e. the A3571-A3572-A3575 chain. In Sect. 2 we give an overview of optical and X-ray properties of the A3571 complex; in Sect. 3 we report on the radio observations; the radio source sample and the optical identifications are described in Sect. 4; the radio properties of the A3571 complex are presented in Sect. 5, and the radio and optical number counts are derived in Sect. 6. A Discussion and conclusions are given in Sect. 7 .

We will use $H_{\mathrm{o}}=100 \mathrm{~km} \mathrm{~s}^{-1} \mathrm{Mpc}^{-1}$ and $q_{\mathrm{o}}=0$, which leads to a linear scale $1 \operatorname{arcsec}=0.55 \mathrm{kpc}$ at the average redshift of the cluster complex $(z=0.039)$.

\section{The A3571 complex in the Shapley Concentration}

The chain formed by the three clusters A3571-A3572A3575 lies $\sim 5^{\circ}$ east of the geometrical centre of the Shapley Concentration, defined by the A3558 complex. In Fig. 1, we plotted the galaxy isodensity contours in this region, after smoothing the optical counts (derived from the COSMOS catalogue) with a Gaussian of 6 arcmin of $F W H M$. The size of the structure is approximately $150 \times 70$ arcmin, corresponding to $5.1 \times 2.4 h^{-1} \mathrm{Mpc}$ at $11550 \mathrm{~km} \mathrm{~s}^{-1}$ (the average velocity of the three clusters). The presence of a significant overdensity at the position of A3571 is evident, with a tail extending down to the centre of A3572. It is difficult to assess if A3572 is part of A3571, or if it is an independent structure. Less clear is the existence of a galaxy excess at the position of A3575.

Table 1 summarizes the most important features of the three clusters: in Cols. 1, 2 and 3 we give respectively the name and J2000 coordinates of the centre (taken from the ACO catalogue, Abell et al. 1989); in Col. 4 the BautzMorgan type and richness class; in Cols. 5 and 6 the average recession velocity and the velocity dispersion respectively.

\section{1. $A 3571$}

The dominant member of the complex is the cluster A3571, with richness class $R=2$ (Abell et al. 1989) and Bautz-Morgan type I. The mean velocity is $v=11730 \pm$ $127 \mathrm{~km} \mathrm{~s}^{-1}$ and the velocity dispersion is $1022_{-77}^{+99} \mathrm{~km} \mathrm{~s}^{-1}$ (Quintana \& de Souza 1993). Quintana \& de Souza (1993)

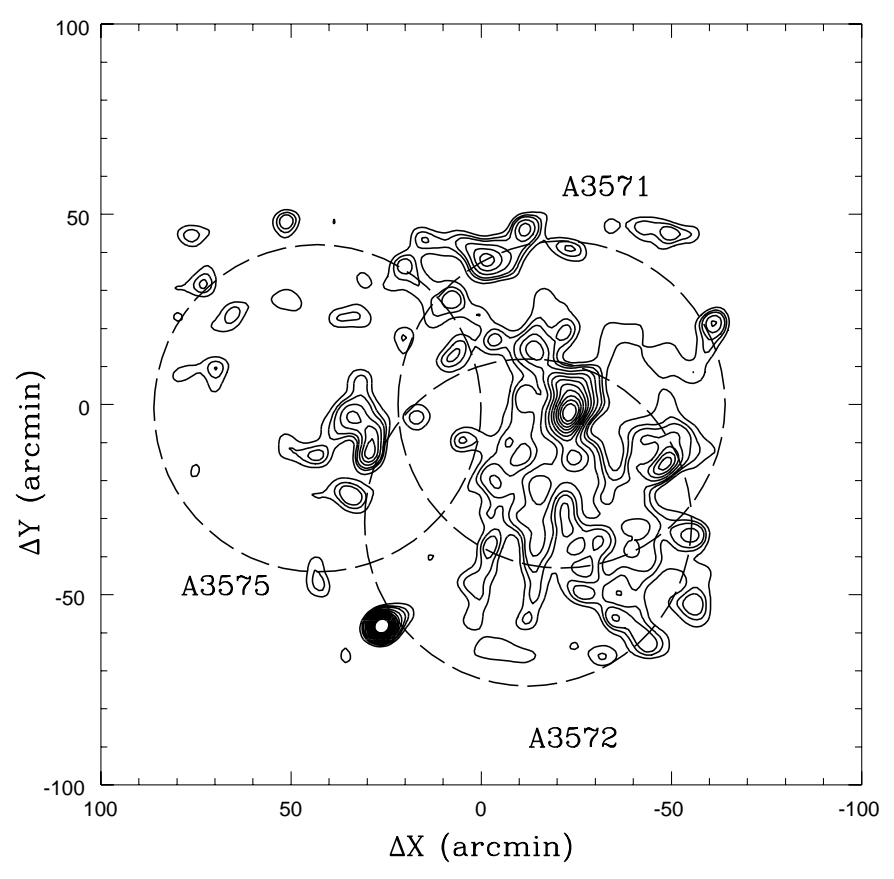

Fig. 1. Isocontours of the galaxy density to $b_{j}=19.5$ in the A3571 complex. The figure is centered at $\alpha=13^{\mathrm{h}} 49^{\mathrm{m}} 10^{\mathrm{s}}, \delta=$ $-32^{\circ} 31^{\prime} 47^{\prime \prime}$. Dashed lines correspond to 1 Abell radius circles around cluster centres, given in Table 1 . The counts have been smoothed with a Gaussian of $6 \operatorname{arcmin} F W H M$.

Table 1. Properties of the clusters in the A3571 chain.

\begin{tabular}{ccccccc}
\hline Cluster & $\begin{array}{c}\mathrm{RA}_{\mathrm{J} 2000} \\
\mathrm{~h}\end{array}$ & $\begin{array}{c}\mathrm{DEC}_{\mathrm{J} 2000} \\
{ }^{\prime}\end{array}$ & $\mathrm{B}-\mathrm{M}(R)$ & $\begin{array}{c}v_{\mathrm{rec}} \\
\mathrm{km} \mathrm{s}^{-1}\end{array}$ & $\begin{array}{c}\sigma_{v} \\
\mathrm{~km} \mathrm{~s}^{-1}\end{array}$ \\
\hline $\mathrm{A} 3571$ & 134730 & -3251 & $\mathrm{I}(2)$ & $11730^{a}$ & $1022^{a}$ \\
$\mathrm{~A} 3572$ & 134812 & -3322 & I-II (0) & $12142^{b}$ & $1300^{b}$ \\
$\mathrm{~A} 3575$ & 135236 & -3252 & II (0) & $11250^{c}$ & $572^{c}$ \\
\hline
\end{tabular}

${ }^{a}$ Quintana \& de Souza (1993).

${ }^{b}$ Drinkwater et al. (1999).

${ }^{c}$ Quintana et al. (1997).

suggested that the velocity distribution is asymmetric and that the bi-dimensional galaxy distribution presents some indications of substructure.

A3571 is characterised by the presence of the large cD galaxy MCG05-33-002, located at its centre. This galaxy, with an extension of $0.1 \times 0.3 \mathrm{~h}^{-1} \mathrm{Mpc}$, is elongated along the north-south direction (Kemp \& Meaburn 1991), in the same direction of the bi-dimensional galaxy distribution.

As noted by Nevalainen et al. (2000, 2001), while the galaxy distribution indicates some degree of nonrelaxation, the $\mathrm{X}$-ray observations show that $\mathrm{A} 3571$ is a well relaxed cluster, with the presence of a cooling flow. The hot gas temperature is $7.6 \mathrm{keV}$ (Nevalainen et al. 2001), consistent with the galaxy velocity dispersion. Nevalainen et al. (2001) determined also the total mass using the hydrostatic equilibrium of the hot gas, and found $M_{\mathrm{tot}} \sim 4.6 \times 10^{14} h^{-1} M_{\odot}$. For comparison, 
Table 2. Details on the observations.

\begin{tabular}{|c|c|c|c|c|}
\hline Field & $\begin{array}{l}\mathrm{RA}_{\mathrm{J} 2000} \\
\mathrm{~h} \mathrm{~m} \text { s }\end{array}$ & $\underset{\circ,}{\mathrm{DEC}_{\mathrm{J} 2000}}$ & $\begin{array}{l}\mathrm{rms}_{22 \mathrm{~cm}} \\
\mathrm{mJy} \mathrm{b}^{-1}\end{array}$ & $\begin{array}{c}\mathrm{rms}_{13 \mathrm{~cm}} \\
\mathrm{mJy} \mathrm{b}^{-1}\end{array}$ \\
\hline$\# 1$ & 134730 & -3251 & 0.12 & 0.12 \\
\hline$\# 2$ & 134911 & -3251 & 0.11 & 0.11 \\
\hline$\# 3$ & 135052 & -3251 & 0.11 & 0.12 \\
\hline$\# 4$ & 135231 & -3251 & 0.12 & 0.12 \\
\hline$\# 5$ & 134757 & -3310 & 0.11 & 0.11 \\
\hline$\# 6$ & 134812 & -3325 & 0.11 & 0.11 \\
\hline
\end{tabular}

the results of the virial mass of Girardi et al. (1998) is $M_{\text {tot }} \sim 9 \times 10^{14} h^{-1} M_{\odot}$.

\section{2. $A 3572$ and $A 3575$}

Little is known about the other two clusters in the complex.

A3572 has a richness class $R=0$ and is located south of A3571 in the plane of the sky (see Fig. 1). Drinkwater et al. (1999) found a mean velocity of $\langle v\rangle=12142 \mathrm{~km} \mathrm{~s}^{-1}$, which implies an average redshift $\langle z\rangle=0.0405$.

A3575 has $R=0$ and lies east of A3571. Quintana et al. (1997) found $\langle v\rangle=11250 \mathrm{~km} \mathrm{~s}^{-1}$ (i.e. $<z>=0.0375)$ and $\sigma=572 \mathrm{~km} \mathrm{~s}^{-1}$ on the basis of only 12 velocities.

No information is available in the literature on the $\mathrm{X}-$ ray properties of these two clusters. Visual inspection of the ROSAT All Sky Survey images does not show any indication of $\mathrm{X}$-ray emission.

\section{Observations and data reduction}

\section{1. $22 \mathrm{~cm}$ and $13 \mathrm{~cm}$ ATCA observations}

We imaged the A3571 complex with the Australia Telescope Compact Array (ATCA) at $22 \mathrm{~cm}(\nu=$ $1.38 \mathrm{GHz})$ and $13 \mathrm{~cm}(\nu=2.38 \mathrm{GHz})$ simultaneously. The whole region was covered with six different pointings, whose centres are listed in Table 2 . The separation between contiguous fields is 20 arcmin.

The observations were carried out in 1995 (December 22 and 23), using the mosaicing facility of the ATCA. To obtain a good hour angle coverage, the six fields were observed in sequence, for two minutes each. The cycle (including calibration) was repeated for $2 \times 12$ hours. The array operated is the $6.0 \mathrm{C}$ configuration (full ATCA resolution), which yields a resolution of $\sim 11^{\prime \prime} \times 5^{\prime \prime}$ at the declination of the A3571 complex. The largest detectable structure size is $\sim 3$ arcmin.

We observed in continuum mode with a $128-\mathrm{MHz}$ bandwidth, divided in $32 \times 4 \mathrm{MHz}$ channels, in order to reduce the bandwidth smearing effects at large distance from the pointing centres. B1934-638 was used as primary flux density calibrator, with assumed flux densities $S_{22 \mathrm{~cm}}=14.9 \mathrm{Jy}$ and $S_{13 \mathrm{~cm}}=11.6 \mathrm{Jy}$.

\subsection{Data reduction and image analysis}

The data reduction was carried out with the package MIRIAD (Sault et al. 1995), which is particularly suited to the ATCA observations. Multifrequency synthesis techniques are implemented, and this allows proper gridding of the data in order to reduce bandwidth smearing effects. This is an important feature, given that we want to image the fields as large as possible. We reduced the data and imaged each field separately. The six cleaned $22 \mathrm{~cm}$ images were then mosaiced (i.e., linearly combined) using the $M I R I A D$ task $L I N M O S$, in order to obtain a uniform sensitivity over the whole region. The area covered by our mosaic is $\sim 2.4 \mathrm{deg}^{2}$. The image analysis was carried out with the AIPS package.

As shown in Fig. 2, a pointing separation of 20 arcmin allows a good overlap between fields at $22 \mathrm{~cm}$, where the primary beam size is $F W H M \sim 33^{\prime}$, and it is best suited to get uniform sensitivity over the central region of the radio mosaic (Prandoni et al. 2000a). On the other hand, the primary beam size at $13 \mathrm{~cm}\left(F W H M \sim 22^{\prime}\right)$ is comparable to the distance between adjacent fields, and the very little overlap does not allow us to compensate for the radial sensitivity loss due to the primary beam attenuation. The mosaic technique is not effective at this frequency, therefore in this case we treated each image separately.

The average rms noise values measured in the individual fields at both $22 \mathrm{~cm}$ and $13 \mathrm{~cm}$ are listed in Table 2; the average rms flux density measured over the whole mosaic is $\sigma=0.13 \mathrm{mJy} \mathrm{b}^{-1}$. At both wavelengths we assumed a source detection flux limit $S=0.65 \mathrm{mJy} \mathrm{b}^{-1}$ (i.e. $\left.\sim 5 \sigma\right)$. Such a limit translates into a radio power detection limit $\log P_{22}\left(\mathrm{~W} \mathrm{~Hz}^{-1}\right)=21.02$ at the average distance of the A3571 cluster complex.

The internal uncertainty of the source flux density and position measures can be estimated following Prandoni et al. (2000b), since our ATCA interferometric data are very similar to those presented in their work (same frequency and array configuration).

For unresolved (pointlike) radio sources the uncertainty $\Delta S$, associated with the flux density, depends on the flux density itself $\left(S_{\text {peak }}\right)$, on the local signal-to-noise ratio, defined as $S_{\text {peak }} / \sigma$, and on the calibration residual errors, as follows:

$\Delta S=\sigma\left(S_{\text {peak }}\right) / S_{\text {peak }}=0.93\left(\frac{S_{\text {peak }}}{\sigma}\right)^{-1}$.

Using this equation, a radio source with peak flux density $S_{\text {peak }}=0.65 \mathrm{mJy} \mathrm{b}^{-1}$ and signal-to-noise ratio of 5 , has $\Delta S=0.12 \mathrm{mJy}$.

The internal position error of the radio sources depends again on the local signal-to-noise ratio, and on the synthesized beam, according to the following formulas (Prandoni et al. 2000b):

$\sigma_{\alpha} \simeq \frac{b_{\min }}{2}\left(\frac{S_{\text {peak }}}{\sigma}\right)^{-1}$, 


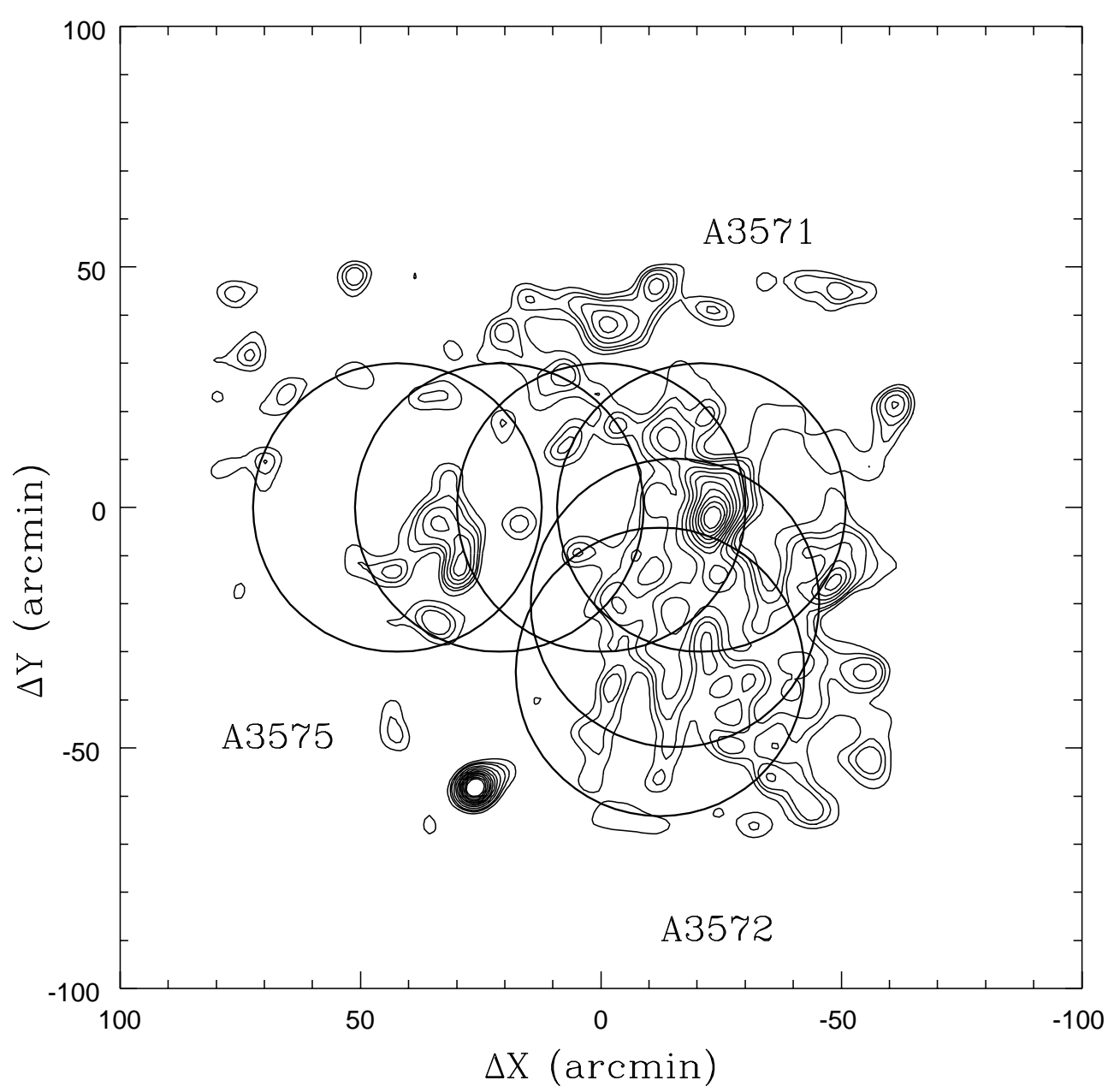

Fig. 2. Fields of the observations overlaid on the the galaxy density (same as in Fig. 1). The radius of each circle is $30^{\prime}$. The centres of each field are given in Table 2 .

$\sigma_{\delta} \simeq \frac{b_{\mathrm{maj}}}{2}\left(\frac{S_{\mathrm{peak}}}{\sigma}\right)^{-1}$

where we assumed $b_{\min }=5^{\prime \prime}$ and $b_{\text {maj }}=11^{\prime \prime}$, the average synthesized beam values of the $22 \mathrm{~cm}$ survey. Radial deformations due to the cromatic aberration are negligible in our mosaic, so we did not compensate for this effect. We assumed for all sources a conservative position uncertainty of $1^{\prime \prime}$, both in RA and in DEC, which corresponds to the values for the weakest sources in the sample, i.e. $5 \sigma$ detections.

\section{Results}

\subsection{The sample of radio sources}

We detected a total of 124 sources at $22 \mathrm{~cm}$ above $0.65 \mathrm{mJy}$ in the mosaic image. We point out that due to the smaller field of view of the $13 \mathrm{~cm}$ observations, and to possible spectral index effects, only a fraction of the sources detected at $22 \mathrm{~cm}$ was revealed at this shorter wavelength. In particular at $13 \mathrm{~cm}$ we detected 36/124 sources, i.e. $29 \%$.

The source list is reported in Table 3, where we give name and J2000 position (Cols. 1, 2 and 3); integrated flux density at $22 \mathrm{~cm}$ corrected for the primary beam attenuation (Col. 4); integrated flux density at $13 \mathrm{~cm}$ corrected for the primary beam attenuation (Col. 5); spectral index $\alpha_{13}^{22}$ for those sources detected at both frequencies (Col. 6), to be read in the sense $S \propto \nu^{-\alpha}$; radio morphology (Col. 7). For those sources undetected at $13 \mathrm{~cm}, \mathrm{a} \star$ is reported in Table 3.

By inspecting our $22 \mathrm{~cm}$ mosaic image, we classified the radio morphology of the sources as follows: unresolved (unres.), resolved/extended (res.) and double (D). For the extended sources in the sample we give the position of the radio peak, while for the two double systems we give the coordinates of the barycentre. The majority of the radio sources, 110 out of $124(\sim 89 \%)$, are unresolved at the resolution of the final image, $2(\sim 2 \%)$ have a double structure and the remaining $12(\sim 9 \%)$ are extended.

In Fig. 3 we show the distribution of the spectral index for those sources detected at both $22 \mathrm{~cm}$ and $13 \mathrm{~cm}$. We point out that the computation of the spectral index was done using the total flux density as measured on the images, so the different $u-v$ coverage at the two wavelengths was not taken into account. For extended/resolved radio sources, this may have biassed our analysis towards spectra steeper than their intrinsic shape, given the lack 
Table 3. Source list and flux density values.

\begin{tabular}{|c|c|c|c|c|c|c|}
\hline Name & $\begin{array}{l}\mathrm{RA}_{\mathrm{J} 2000} \\
\mathrm{~h} \mathrm{~m} \quad \mathrm{~s}\end{array}$ & $\begin{array}{l}\mathrm{DEC}_{\mathrm{J} 2000} \\
\circ \quad, \quad \prime\end{array}$ & $\begin{array}{r}S_{22 \mathrm{~cm}} \\
\mathrm{mJy}\end{array}$ & $\begin{array}{r}S_{13 \mathrm{~cm}} \\
\mathrm{mJy}\end{array}$ & $\alpha_{13}^{22}$ & Morph. \\
\hline J1346-3259 & 134613.0 & -325933 & 2.41 & $\star$ & & unres. \\
\hline J1346-3252 & 134613.7 & -325228 & 3.90 & $\star$ & & unres. \\
\hline J1346-3231 & 134625.9 & -323139 & 5.57 & - & & unres. \\
\hline J1346-3237 & 134634.3 & -323708 & 1.89 & $\star$ & & unres. \\
\hline J1346-3235 & 134638.8 & -323555 & 12.59 & $\star$ & & unres. \\
\hline J1346-3303 & 134639.4 & -330344 & 1.04 & $\star$ & & unres. \\
\hline J1346-3254 & 134640.9 & -325423 & 1.20 & $\star$ & & unres. \\
\hline J1346-3305 & 134647.6 & -330503 & 4.45 & $\star$ & & unres. \\
\hline J1346-3320 & 134650.3 & -332015 & 1.09 & $\star$ & & unres. \\
\hline J1346-3304 & 134650.4 & -330459 & 4.20 & $\star$ & & unres. \\
\hline J1346-3248 & 134653.4 & -324822 & 1.69 & $\star$ & & unres. \\
\hline J1346-3346 & 134659.0 & -334616 & 4.47 & - & & unres. \\
\hline $\mathrm{J} 1347-3311 \mathrm{a}$ & 134702.7 & -331151 & 0.89 & $\star$ & & unres. \\
\hline J1347-3332 & 134707.3 & -333206 & 2.32 & $\star$ & & res. \\
\hline $\mathrm{J} 1347-3339 \mathrm{a}$ & 134708.1 & -333945 & 5.52 & - & & unres. \\
\hline J1347-3306 & 134709.0 & -330646 & 0.84 & $\star$ & & unres. \\
\hline $\mathrm{J} 1347-3313$ & 134709.4 & -331351 & 1.35 & $\star$ & & unres. \\
\hline J1347-3334 & 134720.2 & -333447 & 2.29 & $\star$ & & unres. \\
\hline $\mathrm{J} 1347-3325$ & 134724.2 & -332511 & 16.29 & 4.55 & 2.34 & res. \\
\hline $\mathrm{J} 1347-3252^{\diamond}$ & 134724.6 & -325215 & 24.23 & 11.23 & 1.41 & $\mathrm{D}$ \\
\hline J1347-3256 & 134725.3 & -325610 & 1.34 & $\star$ & & unres. \\
\hline J1347-3324 & 134725.4 & -332441 & 7.08 & $\star$ & & res. \\
\hline J1347-3229 & 134726.5 & -322930 & 54.39 & - & & unres. \\
\hline J1347-3314 & 134726.8 & -331433 & 1.04 & $\star$ & & unres. \\
\hline J1347-3251 & 134728.5 & -325154 & 4.05 & 2.52 & 0.87 & unres. \\
\hline $\mathrm{J} 1347-3310$ & 134731.4 & -331004 & 3.83 & 2.80 & 0.57 & unres. \\
\hline $\mathrm{J} 1347-3241$ & 134734.3 & -324131 & 2.96 & 1.54 & 1.20 & unres. \\
\hline J1347-3323 & 134736.3 & -332328 & 3.38 & 1.92 & 1.04 & unres. \\
\hline J1347-3339b & 134737.3 & -333927 & 7.13 & 7.76 & -0.16 & unres. \\
\hline $\mathrm{J} 1347-3311 \mathrm{~b}$ & 134742.4 & -331143 & 15.60 & 9.15 & 0.98 & unres. \\
\hline J1347-3328 & 134748.7 & -332840 & 4.12 & 1.90 & 1.42 & unres. \\
\hline J1347-3259 & 134753.1 & -325901 & 1.08 & $\star$ & & unres. \\
\hline J1347-3236 & 134754.2 & -323659 & 14.18 & 10.44 & 0.56 & unres. \\
\hline J1347-3301 & 134756.8 & -330106 & 9.09 & 6.12 & 0.73 & unres. \\
\hline $\mathrm{J} 1347-3330^{\circ}$ & 134757.5 & -333002 & 5.06 & 3.79 & 0.53 & unres. \\
\hline J1347-3302 & 134759.5 & -330203 & 0.98 & $\star$ & & unres. \\
\hline J1348-3311 & 134804.2 & -331133 & 1.13 & 0.74 & 0.78 & unres. \\
\hline J1348-3315 & 134804.9 & -331558 & 1.47 & $\star$ & & unres. \\
\hline J1348-3246 & 134808 & -324615 & 192.08 & 102.54 & 1.15 & $\mathrm{D}$ \\
\hline J1348-3339 & 134810.5 & -333931 & 2.25 & $\star$ & & unres. \\
\hline J1348-3323 & 134814.3 & -332332 & 2.22 & $\star$ & & unres. \\
\hline J1348-3239 & 134815.3 & -323931 & 1.04 & $\star$ & & unres. \\
\hline J1348-3255 & 134816.1 & -325533 & 0.75 & $\star$ & & unres. \\
\hline J1348-3234 & 134824.9 & -323435 & 4.06 & $\star$ & & unres. \\
\hline J1348-3249 & 134830.3 & -324910 & 1.03 & $\star$ & & unres. \\
\hline J1348-3256 & 134832.3 & -325633 & 1.82 & $\star$ & & unres. \\
\hline J1348-3318 & 134833.5 & -331847 & 0.92 & $\star$ & & unres. \\
\hline J1348-3328 & 134835.1 & -332818 & 0.69 & $\star$ & & unres. \\
\hline J1348-3310 & 134837.5 & -331004 & 11.70 & 6.75 & 1.01 & unres. \\
\hline J1348-3258 & 134839.6 & -325816 & 1.22 & 1.46 & -0.33 & unres. \\
\hline
\end{tabular}


Table 3. continued.

\begin{tabular}{|c|c|c|c|c|c|c|}
\hline Name & $\begin{array}{l}\mathrm{RA}_{\mathrm{J} 2000} \\
\mathrm{~h} \mathrm{~m} \quad \mathrm{~s}\end{array}$ & $\begin{array}{l}\mathrm{DEC}_{\mathrm{J} 2000} \\
\circ,{ }^{\prime \prime}\end{array}$ & $\begin{array}{r}S_{22 \mathrm{~cm}} \\
\mathrm{mJy}\end{array}$ & $\begin{array}{r}S_{13 \mathrm{~cm}} \\
\mathrm{mJy}\end{array}$ & $\alpha_{13}^{22}$ & Morph. \\
\hline $\mathrm{J} 1348-3257$ & 134846.3 & -325737 & 6.42 & 4.04 & 0.85 & unres. \\
\hline J1348-3241 & 134846.5 & -324141 & 4.35 & 3.37 & 0.47 & res. \\
\hline J1348-3303 & 134847.7 & -330316 & 14.15 & 6.52 & 1.42 & unres. \\
\hline J1348-3305 & 134852.9 & -330517 & 3.22 & * & & unres. \\
\hline J1348-3304 & 134855.6 & -330459 & 3.74 & $\star$ & & unres. \\
\hline J1348-3250 & 134858.9 & -325045 & 0.76 & $\star$ & & unres. \\
\hline J1348-3337 & 134859.8 & -333755 & 2.68 & 夫 & & unres. \\
\hline J1349-3326 & 134902.3 & -332657 & 0.93 & 夫 & & unres. \\
\hline J1349-3243 & 134907.8 & -324336 & 3.00 & 夫 & & res. \\
\hline J1349-3320a & 134910.8 & -332012 & 3.70 & $\star$ & & unres. \\
\hline J1349-3317 & 134915.0 & -331750 & 2.82 & $\star$ & & unres. \\
\hline J1349-3304 & 134915.6 & -330443 & 3.27 & 2.39 & 0.58 & unres. \\
\hline J1349-3247 & 134918.8 & -324728 & 1.93 & 0.89 & 1.42 & unres. \\
\hline $\mathrm{J} 1349-3314 \mathrm{a}$ & 134923.9 & -331408 & 5.83 & * & & unres. \\
\hline J1349-3323 & 134926.0 & -332356 & 23.95 & $\star$ & & unres. \\
\hline J1349-3329 & 134927.0 & -332927 & 0.73 & 夫 & & unres. \\
\hline J1349-3246 & 134927.7 & -324611 & 1.13 & $\star$ & & unres. \\
\hline $\mathrm{J} 1349-3230$ & 134927.9 & -323010 & 1.72 & - & & unres. \\
\hline $\mathrm{J} 1349-3238 \mathrm{a}$ & 134931.5 & -323806 & 1.59 & - & & unres. \\
\hline J1349-3351 & 134935.1 & -335104 & 212.79 & - & & unres. \\
\hline $\mathrm{J} 1349-3314 \mathrm{~b}$ & 134936.1 & -331434 & 10.28 & - & & unres. \\
\hline J1349-3301 & 134940.5 & -330119 & 2.66 & $\star$ & & unres. \\
\hline $\mathrm{J} 1349-3314 \mathrm{c}$ & 134940.6 & -331428 & 9.32 & - & & unres. \\
\hline J1349-3244 & 134940.8 & -324438 & 1.92 & $\star$ & & unres. \\
\hline $\mathrm{J} 1349-3320 \mathrm{~b}$ & 134943.1 & -332018 & 14.86 & - & & unres. \\
\hline $\mathrm{J} 1349-3238 \mathrm{~b}$ & 134958.2 & -323849 & 5.56 & 7.35 & -0.51 & unres. \\
\hline J1349-3245 & 134958.9 & -324504 & 7.02 & 4.55 & 0.80 & unres. \\
\hline J1350-3334 & 135001.2 & -333422 & 10.00 & - & & unres. \\
\hline J1350-3302 & 135008.8 & -330219 & 4.37 & $\star$ & & res. \\
\hline J1350-3311 & 135009.1 & -331124 & 6.01 & - & & unres. \\
\hline J1350-3301 & 135012.1 & -330158 & 1.21 & $\star$ & & unres. \\
\hline J1350-3249 & 135018.7 & -324917 & 2.55 & 1.66 & 0.79 & unres. \\
\hline J1350-3336 & 135022.5 & -333658 & 56.99 & - & & unres. \\
\hline J1350-3243 & 135026.8 & -324338 & 9.22 & 6.16 & 0.74 & unres. \\
\hline $\mathrm{J} 1350-3248 \mathrm{a}$ & 135046.3 & -324851 & 1.35 & $\star$ & & unres. \\
\hline $\mathrm{J} 1350-3250$ & 135051.7 & -325017 & 1.08 & 0.91 & 0.31 & unres. \\
\hline $\mathrm{J} 1350-3248 \mathrm{~b}$ & 135057.1 & -324848 & 1.07 & $\star$ & & unres. \\
\hline $\mathrm{J} 1351-3248$ & 135124.6 & -324849 & 2.06 & $\star$ & & unres. \\
\hline J1351-3238 & 135128.4 & -323835 & 0.91 & $\star$ & & unres. \\
\hline $\mathrm{J} 1351-3259 \mathrm{a}$ & 135129.4 & -325911 & 1.50 & $\star$ & & unres. \\
\hline J1351-3249 & 135133.8 & -324949 & 2.80 & 1.31 & 1.39 & unres. \\
\hline $\mathrm{J} 1351-3228 \mathrm{a}$ & 135134.1 & -322844 & 32.75 & - & & unres. \\
\hline $\mathrm{J} 1351-3228 \mathrm{~b}$ & 135135.5 & -322845 & 57.82 & - & & unres. \\
\hline J1351-3234 & 135135.5 & -323413 & 3.42 & - & & unres. \\
\hline J1351-3247 & 135135.9 & $-3247 \quad 10$ & 3.14 & $\star$ & & res. \\
\hline $\mathrm{J} 1351-3259 \mathrm{~b}$ & 135141.6 & -325911 & 3.29 & 夫 & & unres. \\
\hline J1351-3313 & 135143.8 & -331348 & 1.67 & - & & unres. \\
\hline $\mathrm{J} 1351-3303$ & 135144.0 & -330307 & 0.94 & $\star$ & & unres. \\
\hline J1351-3310 & 135146.2 & -331042 & 2.79 & - & & unres. \\
\hline J1351-3301 & 135156.9 & -330146 & 1.60 & $\star$ & & unres. \\
\hline
\end{tabular}


Table 3. continued.

\begin{tabular}{|c|c|c|c|c|c|c|}
\hline Name & $\begin{array}{l}\mathrm{RA}_{\mathrm{J} 2000} \\
\mathrm{~h} \mathrm{~m} \mathrm{~s}\end{array}$ & $\begin{array}{l}\mathrm{DEC}_{\mathrm{J} 2000} \\
\circ,{ }^{\prime \prime}\end{array}$ & $\begin{array}{r}S_{22 \mathrm{~cm}} \\
\mathrm{mJy}\end{array}$ & $\begin{array}{r}S_{13 \mathrm{~cm}} \\
\mathrm{mJy}\end{array}$ & $\alpha_{13}^{22}$ & Morph. \\
\hline J1351-3240 & 135159.8 & $\begin{array}{lll}-32 & 40 & 19\end{array}$ & 2.02 & $\star$ & & unres. \\
\hline J1351-3229 & 135159.9 & -322924 & 2.94 & - & & unres. \\
\hline J1352-3314 & 135208.0 & -331445 & 20.42 & - & & res. \\
\hline $\mathrm{J} 1352-3315$ & 135209.1 & -331526 & 26.16 & - & & res. \\
\hline J1352-3236 & 135214.2 & -323644 & 1.22 & $\star$ & & unres. \\
\hline $\mathrm{J} 1352-3243 \mathrm{a}$ & 135216.5 & -324338 & 1.24 & $\star$ & & unres. \\
\hline $\mathrm{J} 1352-3245$ & 135224.8 & -324537 & 12.42 & 6.49 & 1.19 & res. \\
\hline J1352-3308 & 135232.9 & -330849 & 2.37 & $\star$ & & unres. \\
\hline J1352-3306 & 135233.1 & -330601 & 6.52 & 2.95 & 1.46 & unres. \\
\hline $\mathrm{J} 1352-3243 \mathrm{~b}$ & 135234.1 & -324332 & 6.56 & 3.51 & 1.15 & unres. \\
\hline J1352-3244 & 135234.9 & -324433 & 11.00 & 6.13 & 1.07 & unres. \\
\hline J1352-3248 & 135240.2 & -324856 & 1.34 & 1.07 & 0.41 & unres. \\
\hline $\mathrm{J} 1352-3230 \mathrm{a}$ & 135242.6 & -323051 & 5.23 & - & & unres. \\
\hline J1352-3302 & 135243.5 & -330229 & 8.13 & 8.92 & -0.17 & unres. \\
\hline $\mathrm{J} 1352-3230 \mathrm{~b}$ & 135246.3 & -323024 & 10.22 & - & & unres. \\
\hline J1352-3301 & 135250.2 & -330158 & 16.70 & 3.32 & 2.96 & res. \\
\hline J1352-3251 & 135250.5 & -325124 & 232.18 & 145.41 & 0.86 & unres. \\
\hline J1352-3304 & 135256.6 & -330440 & 37.85 & 27.57 & 0.58 & unres. \\
\hline J1353-3229 & 135308.4 & -322927 & 5.81 & - & & unres. \\
\hline J1353-3228 & 135320.1 & -322803 & 6.00 & - & & unres. \\
\hline J1353-3238 & 135322.5 & -323811 & 1.44 & $\star$ & & unres. \\
\hline J1353-3239 & 135327.2 & -323917 & 4.68 & $\star$ & & unres. \\
\hline J1353-3300 & 135345.5 & -330001 & 4.18 & $\star$ & & unres. \\
\hline J1354-3251 & 135424.1 & -325102 & 14.66 & - & & res. \\
\hline
\end{tabular}

$\star$ Non detections at $13 \mathrm{~cm}$, i.e., $S_{13} \leq 0.65 \mathrm{mJy}$ in the image not corrected for the primary beam attenuation.

$\diamond$ The position of this radio source was taken from the $13 \mathrm{~cm}$ image, where the nucleus of the double structure is clearly visible.

* This source shows a double morphology in the $13 \mathrm{~cm}$ image.

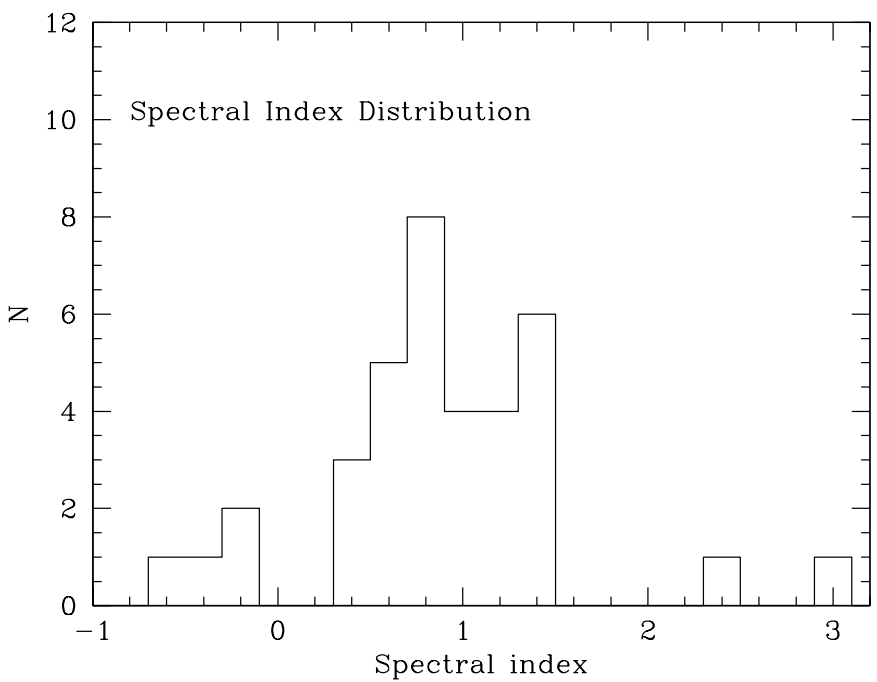

Fig. 3. Distribution of the spectral index $\alpha_{13}^{22}$ for those sources in the sample detected at both wavelengths.

of short spacings at $13 \mathrm{~cm}$. This effect is particularly evident for the two sources with the steepest spectra: they are both extended sources (see Table 3), and we believe that a fraction of their extended flux was missed at $13 \mathrm{~cm}$.
From Fig. 3 we conclude that the sample of radio sources includes both compact active nuclei with flat and inverted spectrum, and radio sources with a normal to moderately steep spectrum.

\subsection{Optical identifications}

We searched for optical counterparts of the radio sources detected in our $22 \mathrm{~cm}$ survey using two different catalogues: the COSMOS/UKST Southern Sky Object Catalogue (Yentis et al. 1992), limited to $b_{j}=$ 19.5, and the APM Catalogue (Automated Photographic Measuring), limited to $b_{j}=20$ (Maddox et al. 1990). Both catalogues are the result of automatic scans of the UK Schimdt Telescope (UKST) plates and have a claimed positional accuracy of $\sim 0.25^{\prime \prime}$. The transformation from the plate frame to the sky, however, introduces further uncertainties, so we adopted a conservative mean positional error of 1.5" (Unewisse et al. 1993).

Due to incompleteness effects at both faint and bright magnitudes (bright galaxies can be resolved out) all radio sources were overplotted on the Digitized Sky Survey (DSS) images and scrutinized by eye, to make sure that no identification was missed. 


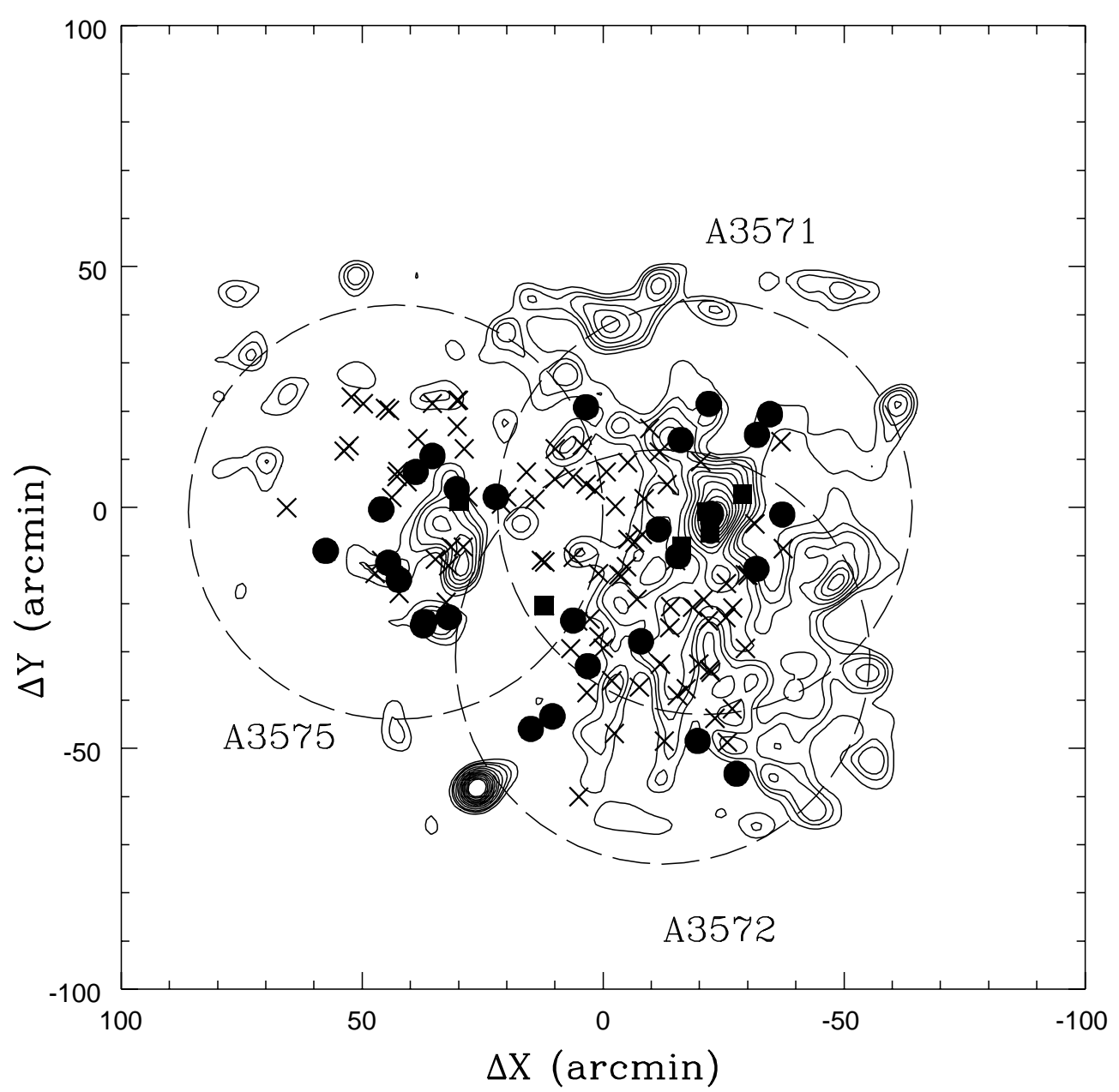

Fig. 4. Location of the radio sources overlaid on the optical isodensities of the A3571 complex. Filled squares represent the optically identified radio galaxies with measured redshift, filled circles those without redshift information and crosses stand for the radio sources without optical counterparts.

Given the uncertainties in the radio and optical positions, we estimated the reliability of our identifications using the parameter $R$, defined as:

$R^{2}=\frac{\Delta_{\mathrm{r}-\mathrm{o}}^{2}}{\sigma_{\mathrm{g}}^{2}+\sigma_{\mathrm{r}}^{2}}$

where $\Delta_{\mathrm{r}-\mathrm{o}}$ is the positional offset between the radio and optical coordinates, $\sigma_{\mathrm{g}}$ is the optical position error and $\sigma_{\mathrm{r}}$ is the uncertainty in the radio position.

For point-like radio sources and point-like optical counterparts, we considered as reliable identifications all matches with $R \leq 3$; for the extended radio sources and/or extended optical galaxies, the value of $R$ is less meaningful and we trusted the direct eye inspection.

Finally, we used NED (NASA Extragalactic Database) to search for the redshift information of the optical counterparts, and considered as belonging to the Shapley Concentration all galaxies with recession velocities in the range $10000<v_{\mathrm{r}}\left(\mathrm{km} \mathrm{s}^{-1}\right)<18000$.

We found 36 optical counterparts, $\sim 29 \%$ of the total, 6 of which with known redshift and all belonging to the A3571 complex ( $17 \%$ of the 36 sources identified). The list of the radio-optical identifications is given in Table 4, which is organised as follows: Col. 1 = radio and optical name (where available); Cols. 2 and $3=$ coordinates (J2000) of the radio source and of the optical counterpart; Col. $4=$ flux density of the radio source at $22 \mathrm{~cm}$ and the $b_{j}$ magnitude of the counterpart; Col. $5=$ flux density at $13 \mathrm{~cm}$; Col. $6=$ spectral index $\alpha_{13}^{22}$ and optical catalogue used to retrieve the information $(\mathrm{A}=\mathrm{APM}, \mathrm{C}=$ COSMOS); Col. $7=$ monochromatic radio power at $22 \mathrm{~cm}$ for the radio galaxies with known redshift and absolute magnitude $B_{j}$ of the optical counterpart; Col. $8=$ radio morphology and parameter $R$; Col. $9=$ cluster where the radio galaxy is located and recession velocity.

In a number of cases the large optical extent of the galaxy and/or the extent of the radio emission lead to $R>3$. For these sources we considered the identification as reliable if the optical counterpart falls within the radio isophotes. A note to Table 4 clarifies these cases.

The location of the radio sources overlaid on the optical isodensities of the A3571 complex is shown in Fig. 4. We note that the radio sources are distributed fairly uniformly over the region covered by our observations, 
Table 4. Optical identifications.

\begin{tabular}{|c|c|c|c|c|c|c|c|c|}
\hline Radio & $\begin{array}{l}\mathrm{RA}_{\mathrm{J} 2000} \\
\mathrm{~h} \quad \mathrm{~m} \mathrm{~s}\end{array}$ & $\begin{array}{l}\text { DEC J2000 }_{0} \\
\circ, \quad \text { " }\end{array}$ & $\begin{array}{r}S_{22 \mathrm{~cm}} \\
\mathrm{mJy}\end{array}$ & $\begin{array}{r}S_{13 \mathrm{~cm}} \\
\mathrm{mJy}\end{array}$ & $\alpha_{13}^{22}$ & $\begin{array}{r}\log P_{22 \mathrm{~cm}} \\
\mathrm{~W} \mathrm{~Hz}\end{array}$ & Radio Type & Cluster \\
\hline Optical & $\begin{array}{l}\mathrm{RA}_{\mathrm{J} 2000} \\
\mathrm{~h} \quad \mathrm{~m} \quad \mathrm{~s}\end{array}$ & $\begin{array}{l}\mathrm{DEC}_{\mathrm{J} 2000} \\
\circ, \quad "\end{array}$ & $b_{j}$ & & Cat. & $B_{j}$ & $R$ & $\begin{array}{c}v_{\mathrm{r}} \\
\mathrm{km} \mathrm{s}^{-1}\end{array}$ \\
\hline J1346-3252 & $\begin{array}{lll}13 & 46 & 13.7 \\
13 & 46 & 13.8\end{array}$ & $\begin{array}{l}-325228 \\
-325226\end{array}$ & $\begin{array}{r}3.90 \\
21.80\end{array}$ & & A & & $\begin{array}{c}\text { unres. } \\
0.90\end{array}$ & \\
\hline J1346-3231 & $\begin{array}{lll}13 & 46 & 25.9 \\
13 & 46 & 25.9\end{array}$ & $\begin{array}{l}-323139 \\
-323139\end{array}$ & $\begin{array}{r}5.57 \\
19.40\end{array}$ & & $\mathrm{C}$ & & $\begin{array}{c}\text { unres. } \\
0.06\end{array}$ & \\
\hline J1346-3237b & $\begin{array}{l}134634.3 \\
134634.3\end{array}$ & $\begin{array}{l}-323708 \\
-323705\end{array}$ & $\begin{array}{r}1.89 \\
18.13\end{array}$ & & $\mathrm{C}$ & & $\begin{array}{c}\text { unres. } \\
1.34\end{array}$ & A3571 \\
\hline J1346-3235 & $\begin{array}{l}134638.8 \\
134638.8\end{array}$ & $\begin{array}{l}-323555 \\
-323554\end{array}$ & $\begin{array}{l}12.59 \\
20.31\end{array}$ & & $\mathrm{C}$ & & $\begin{array}{l}\text { res. } \\
0.65\end{array}$ & \\
\hline J1346-3303 & 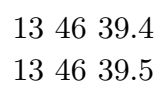 & $\begin{array}{l}-330344 \\
-330344\end{array}$ & $\begin{array}{r}1.04 \\
17.78\end{array}$ & & $\mathrm{C}$ & & $\begin{array}{c}\text { unres. } \\
1.04\end{array}$ & A 3571 \\
\hline J1346-3248 & $\begin{array}{l}134653.4 \\
134653.3\end{array}$ & $\begin{array}{l}-324822 \\
-324820\end{array}$ & $\begin{array}{r}1.69 \\
19.95\end{array}$ & & $\mathrm{C}$ & & $\begin{array}{l}\text { res. } \\
0.99\end{array}$ & \\
\hline J1346-3346 & $\begin{array}{l}134659.0 \\
134658.9\end{array}$ & $\begin{array}{l}-334616 \\
-334612\end{array}$ & $\begin{array}{r}4.47 \\
12.51^{a}\end{array}$ & & $\mathrm{C}$ & & $\begin{array}{c}\text { unres. } \\
1.60\end{array}$ & \\
\hline $\mathrm{J} 1347-3252$ & $\begin{array}{ll}13 & 4724.6 \\
13 & 4724.3\end{array}$ & $\begin{array}{l}-325215 \\
-325213\end{array}$ & $\begin{array}{r}24.23 \\
21.98 ?\end{array}$ & 11.23 & $\begin{array}{c}1.41 \\
\mathrm{C}\end{array}$ & & $\begin{array}{c}\mathrm{D} \\
1.00^{b}\end{array}$ & \\
\hline $\mathrm{J} 1347-3256$ & $\begin{array}{l}134725.3 \\
134725.3\end{array}$ & $\begin{array}{l}-325610 \\
-325613\end{array}$ & $\begin{array}{r}1.34 \\
15.78\end{array}$ & & $\mathrm{C}$ & $\begin{array}{r}21.35 \\
-19.59\end{array}$ & $\begin{array}{c}\text { unres. } \\
1.57\end{array}$ & $\begin{array}{l}\text { A } 3571 \\
11850\end{array}$ \\
\hline J1347-3229 & $\begin{array}{ll}13 & 4726.5 \\
13 & 4726.5\end{array}$ & $\begin{array}{l}-322930 \\
-322930\end{array}$ & $\begin{array}{l}54.39 \\
20.42\end{array}$ & & $\mathrm{C}$ & & $\begin{array}{l}\text { res. } \\
0.08\end{array}$ & \\
\hline $\mathrm{J} 1347-3251$ & $\begin{array}{ll}13 & 4728.5 \\
13 & 4728.3\end{array}$ & $\begin{array}{l}-325154 \\
-325154\end{array}$ & $\begin{array}{r}4.05 \\
13.81\end{array}$ & 2.52 & $\begin{array}{c}0.87 \\
\mathrm{C}\end{array}$ & $\begin{array}{r}21.82 \\
-21.53\end{array}$ & $\begin{array}{c}\text { unres. } \\
1.00\end{array}$ & $\begin{array}{l}\text { A } 3571 \\
11669\end{array}$ \\
\hline J1347-3339 & 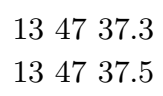 & $\begin{array}{l}-333927 \\
-333930\end{array}$ & $\begin{array}{r}7.13 \\
22.46\end{array}$ & 7.76 & -0.16 & & $\begin{array}{l}\text { res. } \\
1.96\end{array}$ & \\
\hline $\mathrm{J} 1347-3259$ & $\begin{array}{ll}13 & 47 \\
13 & 47.1 \\
13 & 53.1\end{array}$ & $\begin{array}{l}-325901 \\
-325902\end{array}$ & $\begin{array}{r}1.08 \\
15.18\end{array}$ & & $\mathrm{C}$ & $\begin{array}{r}21.20 \\
-20.03\end{array}$ & $\begin{array}{c}\text { unres. } \\
0.32\end{array}$ & $\begin{array}{l}\text { A } 3571 \\
11100\end{array}$ \\
\hline $\mathrm{J} 1347-3236$ & $\begin{array}{l}134754.2 \\
134754.2\end{array}$ & $\begin{array}{l}-323659 \\
-323635\end{array}$ & $\begin{array}{l}14.18 \\
19.77\end{array}$ & 10.44 & $\begin{array}{c}0.56 \\
\mathrm{C}\end{array}$ & & $\begin{array}{c}\text { unres. } \\
0.08\end{array}$ & A 3571 \\
\hline $\mathrm{J} 1347-3301$ & $\begin{array}{l}134756.8 \\
134756.7\end{array}$ & $\begin{array}{l}-330106 \\
-330109\end{array}$ & $\begin{array}{r}9.09 \\
17.98\end{array}$ & 6.12 & $\begin{array}{c}0.73 \\
\mathrm{~A}\end{array}$ & & $\begin{array}{c}\text { unres. } \\
1.33\end{array}$ & \\
\hline J1348-3255 & $\begin{array}{lll}13 & 48 & 16.1 \\
13 & 48 & 16.2\end{array}$ & $\begin{array}{l}-325533 \\
-325533\end{array}$ & $\begin{array}{r}0.75 \\
21.77\end{array}$ & & A & & $\begin{array}{c}\text { unres. } \\
0.20\end{array}$ & \\
\hline
\end{tabular}

regardless of the underlying optical density, which varies considerably going from A3571 to the other clusters in the chain. From Table 4 it is clear that the Shapley radio galaxies, i.e. galaxies with measured redshift, are located in A3571 (4/6) and in the region between the centre of A3571 and A3575 (2/6).

\section{Radio properties of the $\mathbf{A} 3571$ cluster complex}

\subsection{The radio galaxies. Starburst or weak AGN?}

From the optical identification procedure discussed in Sect. 4.2, only six radio sources are found to be associated with galaxies in the Shapley Concentration. We believe this number is underestimated due to the poor spectroscopic information available in this region. Inspection of Table 4 shows that nine radio sources are associated with galaxies brighter than $b_{J} \leq 18.5$ without redshift information. They are located in A3571 and in A3575. It is most likely that at least a fraction of them belongs to the A3571 complex, and in the following we will refer to these objects as "candidate radio galaxies" of the A3571 cluster complex. We note that no radio sources are found to be associated (or possibly associated) with galaxies in the central region of A3572. 
Table 4. continued.

\begin{tabular}{|c|c|c|c|c|c|c|c|c|}
\hline Radio & $\begin{array}{l}\mathrm{RA}_{\mathrm{J} 2000} \\
\mathrm{~h} \quad \mathrm{~m} \quad \mathrm{~s}\end{array}$ & $\begin{array}{l}\mathrm{DEC}_{\mathrm{J} 2000} \\
\circ,{ }^{\prime \prime}\end{array}$ & $\begin{array}{r}S_{22 \mathrm{~cm}} \\
\mathrm{mJy}\end{array}$ & $\begin{array}{r}S_{13 \mathrm{~cm}} \\
\mathrm{mJy}\end{array}$ & $\alpha_{13}^{22}$ & $\begin{array}{r}\log P_{22 \mathrm{~cm}} \\
\mathrm{WHz}\end{array}$ & Radio Type & Cluster \\
\hline Optical & $\begin{array}{l}\mathrm{RA}_{\mathrm{J} 2000} \\
\mathrm{~h} \mathrm{~m} \quad \mathrm{~s}\end{array}$ & $\begin{array}{l}\mathrm{DEC}_{\mathrm{J} 2000} \\
\circ,{ }^{\prime \prime}\end{array}$ & $b_{j}$ & & Cat. & $B_{j}$ & $R$ & $\begin{array}{c}v_{\mathrm{r}} \\
\mathrm{km} \mathrm{s}^{-1}\end{array}$ \\
\hline J1348-3318 & $\begin{array}{l}134833.5 \\
134833.5\end{array}$ & $\begin{array}{l}-331847 \\
-331848\end{array}$ & $\begin{array}{r}0.92 \\
18.57\end{array}$ & & $\mathrm{C}$ & & $\begin{array}{c}\text { unres. } \\
0.60\end{array}$ & \\
\hline J1349-3243 & $\begin{array}{lll}13 & 49 & 07.8 \\
13 & 49 & 07.7\end{array}$ & $\begin{array}{l}-324336 \\
-324334\end{array}$ & $\begin{array}{r}3.00 \\
15.24\end{array}$ & & $\mathrm{C}$ & $\begin{array}{r}21.83 \\
-20.48\end{array}$ & $\begin{array}{l}\text { res. } \\
1.10\end{array}$ & $\begin{array}{c}\text { A3571 } \\
13900\end{array}$ \\
\hline J1349-3323 & $\begin{array}{lll}13 & 49 & 26.0 \\
13 & 49 & 25.9\end{array}$ & $\begin{array}{l}-332356 \\
-332349\end{array}$ & $\begin{array}{l}23.95 \\
15.74\end{array}$ & & $\mathrm{C}$ & & $\begin{array}{l}\text { unres. } \\
3.45^{c}\end{array}$ & A3571/2 \\
\hline J1349-3230 & $\begin{array}{lll}13 & 49 & 27.9 \\
13 & 49 & 27.7\end{array}$ & $\begin{array}{l}-323010 \\
-323011\end{array}$ & $\begin{array}{r}1.72 \\
16.33\end{array}$ & & $\mathrm{C}$ & & $\begin{array}{c}\text { unres. } \\
0.93\end{array}$ & A3571/5 \\
\hline $\mathrm{J} 1349-3314 \mathrm{c}$ & $\begin{array}{l}134940.6 \\
134940.6\end{array}$ & $\begin{array}{l}-331428 \\
-331428\end{array}$ & $\begin{array}{r}9.32 \\
19.40\end{array}$ & & $\mathrm{C}$ & & $\begin{array}{l}\text { res. } \\
0.30\end{array}$ & \\
\hline J1350-3334 & $\begin{array}{lll}13 & 50 & 01.2 \\
13 & 50 & 01.3\end{array}$ & $\begin{array}{l}-333422 \\
-333429\end{array}$ & $\begin{array}{l}10.00 \\
20.06\end{array}$ & & $\mathrm{C}$ & & $\begin{array}{l}\text { res. } \\
3.22^{c}\end{array}$ & \\
\hline J1350-3311 & $\begin{array}{lll}13 & 50 & 09.1 \\
13 & 50 & 09.1\end{array}$ & $\begin{array}{lll}-33 & 1124 \\
-33 & 1123\end{array}$ & $\begin{array}{r}6.01 \\
17.43\end{array}$ & & $\mathrm{C}$ & $\begin{array}{r}22.35 \\
-18.80\end{array}$ & $\begin{array}{c}\text { unres. } \\
0.79\end{array}$ & $\begin{array}{c}\text { A3575 } \\
17688\end{array}$ \\
\hline J1350-3336 & $\begin{array}{l}135022.5 \\
135022.4\end{array}$ & $\begin{array}{l}-333658 \\
-333658\end{array}$ & $\begin{array}{l}56.99 \\
21.53\end{array}$ & & $\mathrm{C}$ & & $\begin{array}{c}\text { unres. } \\
0.66\end{array}$ & \\
\hline $\mathrm{J} 1350-3248 \mathrm{~b}$ & $\begin{array}{lll}13 & 50 & 57.1 \\
13 & 50 & 57.1\end{array}$ & $\begin{array}{l}-324848 \\
-324847\end{array}$ & $\begin{array}{r}1.07 \\
18.33\end{array}$ & & $\mathrm{C}$ & & $\begin{array}{c}\text { unres. } \\
0.44\end{array}$ & A3575 \\
\hline J1351-3249 & $\begin{array}{l}135133.8 \\
135133.8\end{array}$ & $\begin{array}{l}-324949 \\
-324949\end{array}$ & $\begin{array}{r}2.80 \\
16.50\end{array}$ & 1.31 & $\begin{array}{c}1.39 \\
\mathrm{C}\end{array}$ & $\begin{array}{r}21.72 \\
-18.99\end{array}$ & $\begin{array}{c}\text { unres. } \\
0.06\end{array}$ & $\begin{array}{c}\text { A3575 } \\
12542\end{array}$ \\
\hline J1351-3247 & $\begin{array}{lll}13 & 51 & 35.9 \\
13 & 51 & 36.1\end{array}$ & $\begin{array}{l}-324710 \\
-324713\end{array}$ & $\begin{array}{r}3.14 \\
18.82\end{array}$ & 1.34 & $\begin{array}{c}1.56 \\
\mathrm{C}\end{array}$ & & $\begin{array}{l}\text { res. } \\
1.93\end{array}$ & \\
\hline $\mathrm{J} 1351-3313$ & $\begin{array}{l}135143.8 \\
135143.7\end{array}$ & $\begin{array}{l}-331348 \\
-331349\end{array}$ & $\begin{array}{r}1.67 \\
18.37\end{array}$ & & $\mathrm{C}$ & & $\begin{array}{c}\text { unres. } \\
0.85\end{array}$ & A3575 \\
\hline J1351-3240 & $\begin{array}{l}135159.8 \\
135159.8\end{array}$ & $\begin{array}{l}-324019 \\
-324019\end{array}$ & $\begin{array}{r}2.02 \\
17.31\end{array}$ & & $\mathrm{C}$ & & $\begin{array}{c}\text { unres. } \\
0.42\end{array}$ & A 3575 \\
\hline J1352-3314 & $\begin{array}{lll}13 & 52 & 08.0 \\
13 & 52 & 07.9 \\
13 & 52 & 07.5\end{array}$ & $\begin{array}{l}-331445 \\
-331452 \\
-331442\end{array}$ & $\begin{array}{l}20.42 \\
18.19 \\
18.50\end{array}$ & & $\begin{array}{l}\mathrm{C} \\
\mathrm{C}\end{array}$ & & $\begin{array}{c}\text { res. } \\
3.36^{c} \\
3.59^{c}\end{array}$ & \\
\hline $\mathrm{J} 1352-3315$ & $\begin{array}{lll}13 & 52 & 09.1 \\
13 & 52 & 09.5\end{array}$ & $\begin{array}{l}-331526 \\
-331533\end{array}$ & $\begin{array}{l}26.16 \\
20.00\end{array}$ & & $\mathrm{C}$ & & $\begin{array}{c}\text { res. } \\
3.93^{c}\end{array}$ & \\
\hline J1352-3243a & $\begin{array}{lll}13 & 52 & 16.5 \\
13 & 52 & 16.5\end{array}$ & $\begin{array}{l}-324338 \\
-324338\end{array}$ & $\begin{array}{r}1.24 \\
17.23\end{array}$ & & $\mathrm{C}$ & & $\begin{array}{c}\text { unres. } \\
0.10\end{array}$ & A3575 \\
\hline J1352-3306 & $\begin{array}{lll}13 & 52 & 33.1 \\
13 & 52 & 33.1\end{array}$ & $\begin{array}{l}-330601 \\
-330600\end{array}$ & $\begin{array}{r}6.52 \\
22.44\end{array}$ & 2.95 & $\begin{array}{c}1.46 \\
\mathrm{C}\end{array}$ & & $\begin{array}{c}\text { unres. } \\
0.57\end{array}$ & \\
\hline J1352-3302 & $\begin{array}{l}135243.5 \\
135243.5\end{array}$ & $\begin{array}{lll}-33 & 02 & 29 \\
-33 & 02 & 29\end{array}$ & $\begin{array}{r}8.13 \\
18.84\end{array}$ & 8.92 & $\begin{array}{c}-0.17 \\
\mathrm{C}\end{array}$ & & $\begin{array}{c}\text { unres. } \\
0.06\end{array}$ & \\
\hline $\mathrm{J} 1352-3251$ & $\begin{array}{l}135250.5 \\
135250.5\end{array}$ & $\begin{array}{l}-325124 \\
-325124\end{array}$ & $\begin{array}{r}232.18 \\
21.86\end{array}$ & 145.41 & $\begin{array}{c}0.86 \\
\mathrm{C}\end{array}$ & & $\begin{array}{c}\text { unres. } \\
0.10\end{array}$ & \\
\hline $\mathrm{J} 1353-3300$ & $\begin{array}{l}135345.5 \\
135345.4\end{array}$ & $\begin{array}{lll}-33 & 00 & 01 \\
-33 & 00 & 03\end{array}$ & $\begin{array}{r}4.18 \\
18.74\end{array}$ & & $\mathrm{C}$ & & $\begin{array}{c}\text { unres. } \\
1.59\end{array}$ & \\
\hline
\end{tabular}

a The optical counterpart is most likely a star.

${ }^{b}$ The optical candidate falls within the radio isocontours of the double structure.

${ }^{c}$ These identifications have $R>3$, however, due to the extent of the radio emission and/or of the optical counterpart, we can consider them reliable since the optical counterpart falls within the radio contours. 
All the optical counterparts (with and without redshift information) are early-type galaxies, with the exception of J1347-3301 and J1349-3243, both associated with a disk galaxy. The radio galaxies in the A3571 region are faint and unresolved by our observations. Their radio power is in the range $21.20 \leq \log P_{22 \mathrm{~cm}}\left(\mathrm{~W} \mathrm{~Hz}{ }^{-1}\right) \leq 22.35$, i.e. much lower than the average transition value between FRI and FRII radio galaxies (Fanaroff \& Riley 1974), i.e. $\log P_{20}\left(\mathrm{~W} \mathrm{~Hz}^{-1}\right) \simeq 24.5$. Similar properties are shared by all the candidate radio galaxies. If we assume that they are located at the average distance of the A3571 complex $(z=$ $0.039)$, only two of them have $\log P_{22}\left(\mathrm{~W} \mathrm{~Hz}^{-1}\right)>22$.

It is believed that for $\log P_{20}\left(\mathrm{~W} \mathrm{~Hz}^{-1}\right)<22$, starburst becomes the dominant mechanism responsible for the radio emission (see for instance Dwarakanath \& Owen 1999). Unfortunately no spectroscopy is available for the candidates in the A3571 complex, therefore it is not possible to establish whether they are indeed starburst galaxies or weak active galactic nuclei, possibly driven by an ADAF nucleus (Narayan \& Yi 1995). Moreover, only a very small fraction of the A3571 radio galaxies and candidates are included in the list of the $13 \mathrm{~cm}$ detections (see Table 4), and no hint to discriminate between starburst and weak AGN can be obtained from the higher resolution $13 \mathrm{~cm}$ images. We suggest that the A3571 complex radio galaxies and candidates with $\log P_{22}\left(\mathrm{~W} \mathrm{~Hz}^{-1}\right)<21.78$ (see Sect. 5.2 for radio power division between AGN and starburst), nine in total, are starburst radio galaxies.

In the following, we present the images of the most interesting sources detected in the A3571 region.

J1347-3251 (see Fig. 5 and Table 4) is a faint radio source associated with the dominant cD galaxy MGC05-33-002. From our image, it is clear that the extension of the radio emission visible on the NRAO VLA Sky Survey (NVSS, Condon et al. 1998), and apparently associated with the cD galaxy, is actually the blend of J1347-3251 itself and the double source J1347-3252.

J1349-3243 (see Fig. 6 and Table 4) is associated with a disk galaxy located between the centres of A3571 and A3575. The radio emission is elongated in the same direction of the optical major axis.

In Fig. 7 the two background sources J1352-3314 and J1352-3315 are shown. Both sources are extended (roughly $30^{\prime \prime}$ and $40^{\prime \prime}$ respectively), and a few objects fall within the radio density contours. For this reason the optical identification is uncertain (see Table 4), and we cannot exclude that they are two components of a double source. They lie at the edge of one of the $22 \mathrm{~cm}$ fields, in a region not covered by the simultaneous $13 \mathrm{~cm}$ observations, so no information is available on their radio spectrum.

\subsection{The radio luminosity function}

In order to investigate if cluster merger has any effect on the formation of radio sources of nuclear origin, it is important to compare the radio luminosity function (RLF) for early type galaxies in merging environments with the

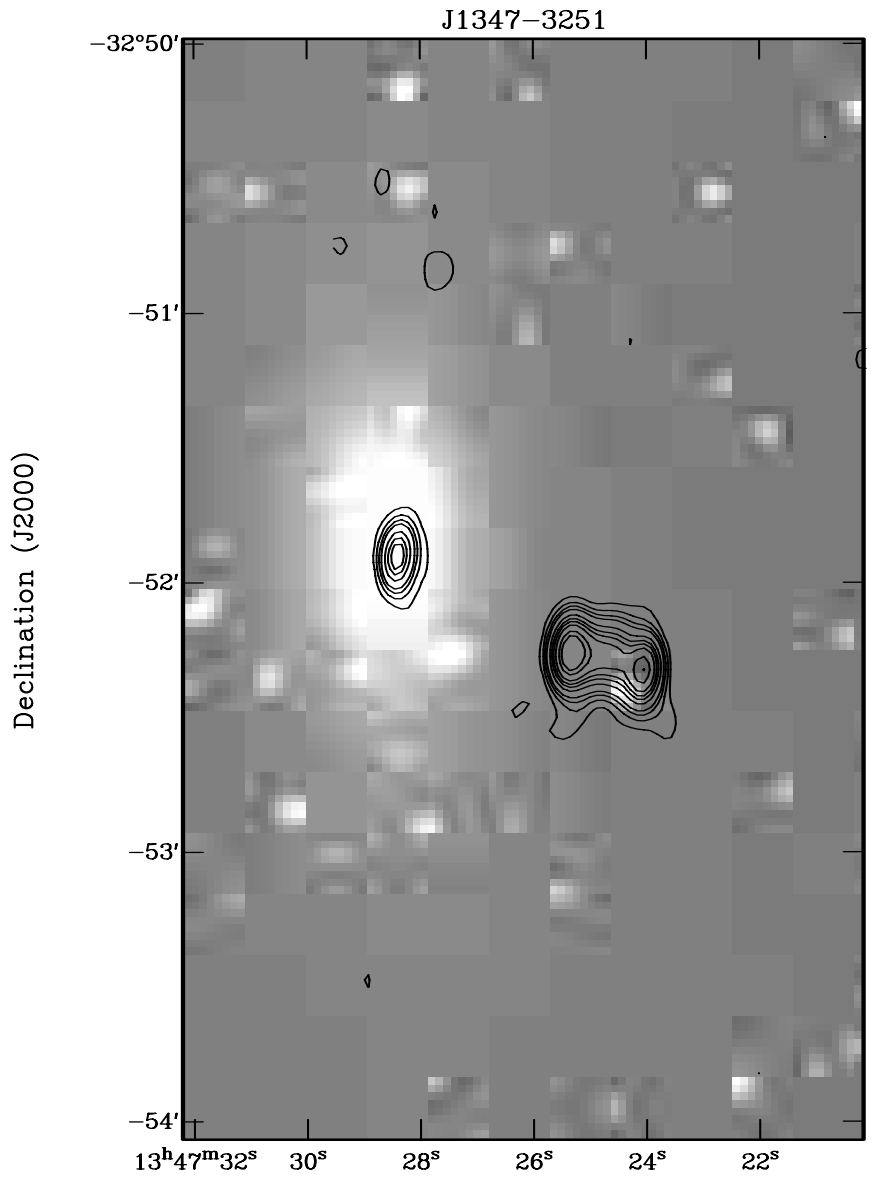

Right Ascension (J2000)

Fig. 5. $22 \mathrm{~cm}$ radio image of J1347-3251 overlaid on the DSS optical frame. The contours are $-0.30,0.30,0.60,0.90,1.20$, $1.90,2.50,3.00,3.70,5.00,6.00 \mathrm{mJy}$. The restoring beam is $11.24^{\prime \prime} \times 5.27^{\prime \prime}$, in PA $-7^{\circ}$. The background double source $\mathrm{J} 1347-3252$ is also visible.

local RLF derived for elliptical galaxies in clusters derived by Ledlow \& Owen (1996, hereinafter LO96).

We carried out this study for the A3571 complex. The limited number of radio galaxies found from our survey, combined with the poor spectroscopic information available in this region of the Shapley Concentration, does not allow a proper determination of the RLF, therefore in the following we will compare the total number of radio galaxies expected on the basis of the RLF derived by LO96, and the number of detections found from our survey. For a proper comparison, we restricted our analysis to those radio galaxies with $\log P_{22}\left(\mathrm{~W} \mathrm{~Hz}^{-1}\right) \geq 21.78$ and with an optical counterpart brighter than $b_{J}=17.70$ (same limits as in LO96, scaled for the proper cosmology and photometric band, see also Venturi et al. 2000).

A number of assumptions were made in order to compensate for the lack of complete spectroscopy in this region, therefore we are aware that the following results should be considered as only indicative. In particular, we assumed that $78 \%$ of the 116 galaxies with $b_{J} \leq 17.70$ in this region, i.e. 90, actually belong to the A3571 complex. 
This fraction was derived for the A3528 and A3558 complexes on the basis of a large number of spectra (Baldi et al. 2001), and we believe that assuming the same percentage also for this nearby cluster complex is a reasonable choice.

From Table 4 we can see that only 3 of the 6 radio galaxies with redshift information have $\log P_{22}\left(\mathrm{~W} \mathrm{~Hz}^{-1}\right) \geq 21.78$. Of the remaining radio sources associated with galaxies brighter than $b_{J}=17.70$ and with no redshift information, only one has $\log P_{22} \geq 21.78$ (J1349-3243) if placed at the distance of the Shapley Concentration (we assumed $z=0.039$ ).

To summarise, the fraction of elliptical galaxies with $b_{J} \leq 17.70$ and $\log P_{22} \geq 21.78$ is $4 / 90(4.4 \%)$, to be compared to the 12 expected ( $14 \%)$ on the basis of LO96. We point out that our estimate should be considered a lower limit, since we implicitly assumed that all 90 galaxies are early type. On the other hand, to obtain the same percentage as in LO96, only $\sim 30 \%$ of the optical galaxies should be early type, a fraction which seems far too low (see Fig. 5 in Baldi et al. 2001). In conclusion, there is an indication that the number of radio galaxies detected over the whole A3571 cluster complex is lower than expected on the basis of the RLF derived in LO96 for cluster ellipticals.

The situation changes considerably if we limit our analysis to the cluster A3571, since all 4 radio galaxies used in the estimate of the RLF are located within 0.5 Abell radius $\left(R_{\mathrm{A}}\right)$ from the centre of $\mathrm{A} 3571$. Using the same arguments illustrated above, the upper limit to the number of elliptical galaxies belonging to A3571 with $b_{J} \leq 17.70$ is 39. The expected number of radio galaxies on the basis of LO96 is 5 , in good agreement with our result. Table 5 summarises the results, and shows the number of radio galaxies expected on the basis of LO96 and the number of detections found in the whole chain and in A3571 only.

\section{Radio and optical source counts}

We derived the radio and source counts in the A3571 complex, in order to check if the optical overdensity in this region of the Shapley supercluster reflects into a higher number of radio sources with respect to the background radio source counts (Prandoni et al. 2001), and for comparison with the radio counts derived for the A3558 and A3528 cluster complexes (Venturi et al. 2000 and 2001 respectively).

Owing to the primary beam attenuation, the sensitivity in our final image drops at the border of the mosaic, so the $22 \mathrm{~cm}$ sample discussed in Sect. 4.1 is not complete to the flux limit of $0.65 \mathrm{mJy}$. For this reason we restricted our analysis to the inner part of the mosaic. In particular, we considered a region of $1.26 \mathrm{deg}^{2}$, where the radio sample is complete down to the flux density of $1.30 \mathrm{mJy}$ and includes 67 radio sources.

The $\log N-\log S$ for the A3571 complex, computed in the flux density range $1.30-593.74 \mathrm{mJy}$, is shown in Fig. 8. The errors are poissonian. We note that the first flux density bin, chosen in the range 1.16-1.64 mJy for comparison
Table 5. Radio luminosity function.

\begin{tabular}{crc}
\hline Region & $\begin{array}{r}N \text { (LO96) } \\
\text { expected }\end{array}$ & $\begin{array}{c}N \\
\text { found }\end{array}$ \\
\hline Whole Chain & 12 & 4 \\
A3571 & 5 & 4 \\
\hline
\end{tabular}

with the background counts, is incomplete. The solid line in Fig. 8 represents the normalized $\log N-\log S$ for the background radio sources at $22 \mathrm{~cm}$ (Prandoni et al. 2001) derived over a sky region covering $25.82 \mathrm{deg}^{2}$.

Figure 8 suggests that the two distributions have very similar shapes. We note that even the point in the first bin is in good agreement with the background counts, despite the fact that it is incomplete.

We quantitatively estimated the similarity between the source counts in the A3571 complex and the background counts applying a Kolmogorov-Smirnov (KS) test to the two distributions. We found that the probability that they are the same distribution is $p=27 \%$. This percentage increases to $85 \%$ if we restrict the comparison to flux density $S_{22 \mathrm{~cm}} \leq 74.22 \mathrm{mJy}$, i.e., dropping the high flux density bins with very few sources. We conclude that the two distributions are statistically indistiguishable. We found 59 radio sources in the flux range 1.64-593.74 mJy (we exclude the incomplete flux density bins). This number is fully consistent with what expected from the background counts, i.e. $61 \pm 6$.

The present result is in agreement with the outcome of our statistical analysis in the A3528 and A3558 cluster complexes, whose radio source counts are also consistent with the background (Venturi et al. 2000, 2001).

For comparison, we estimated the overdensity of the optical galaxies brighter than $m_{\mathrm{b}}=19.5$, with respect to the local background density. We note that for the A3571 region, $m_{\mathrm{b}}=19.5$ becomes $m_{\mathrm{b}}=19.3$ after correcting for the absorption, which is on average $\sim 0.20$.

According to estimates of the optical background, made by Metcalfe et al. (1994) and by Bardelli et al. (1998) in a region of the Shapley Concentration without clusters, the projected overdensity of galaxies $\mathcal{O}$ with $m_{\mathrm{b}}<19.3$ in the A3571 complex is the range $1.39 \leq \mathcal{O}_{\mathcal{B}} \leq 1.78$, depending on the extension of the region considered. This indicates that the number of galaxies in the A3571 complex is on average $50 \%$ above the number expected on the basis of the background optical counts.

If we assume that the probability of radio emission is the same for a field galaxy and for a Shapley galaxy, we would expect a similar excess also in the radio counts, i.e. 92 radio sources for an excess ratio of 1.5 . This excess would be detectable at the $2.5 \sigma$ level, where $\sigma=\left(N_{\text {est }}\right.$ $\left.N_{\text {bck }}\right) \times\left(N_{\text {est }}+N_{\text {bck }}\right)^{-\frac{1}{2}}$. In particular, $N_{\text {est }}=$ estimated number of radio sources and $N_{\mathrm{bck}}=61$, as seen above.

This is in contrast with the result obtained in the radio band, where we showed that the difference in the radio source counts and in the background counts is 


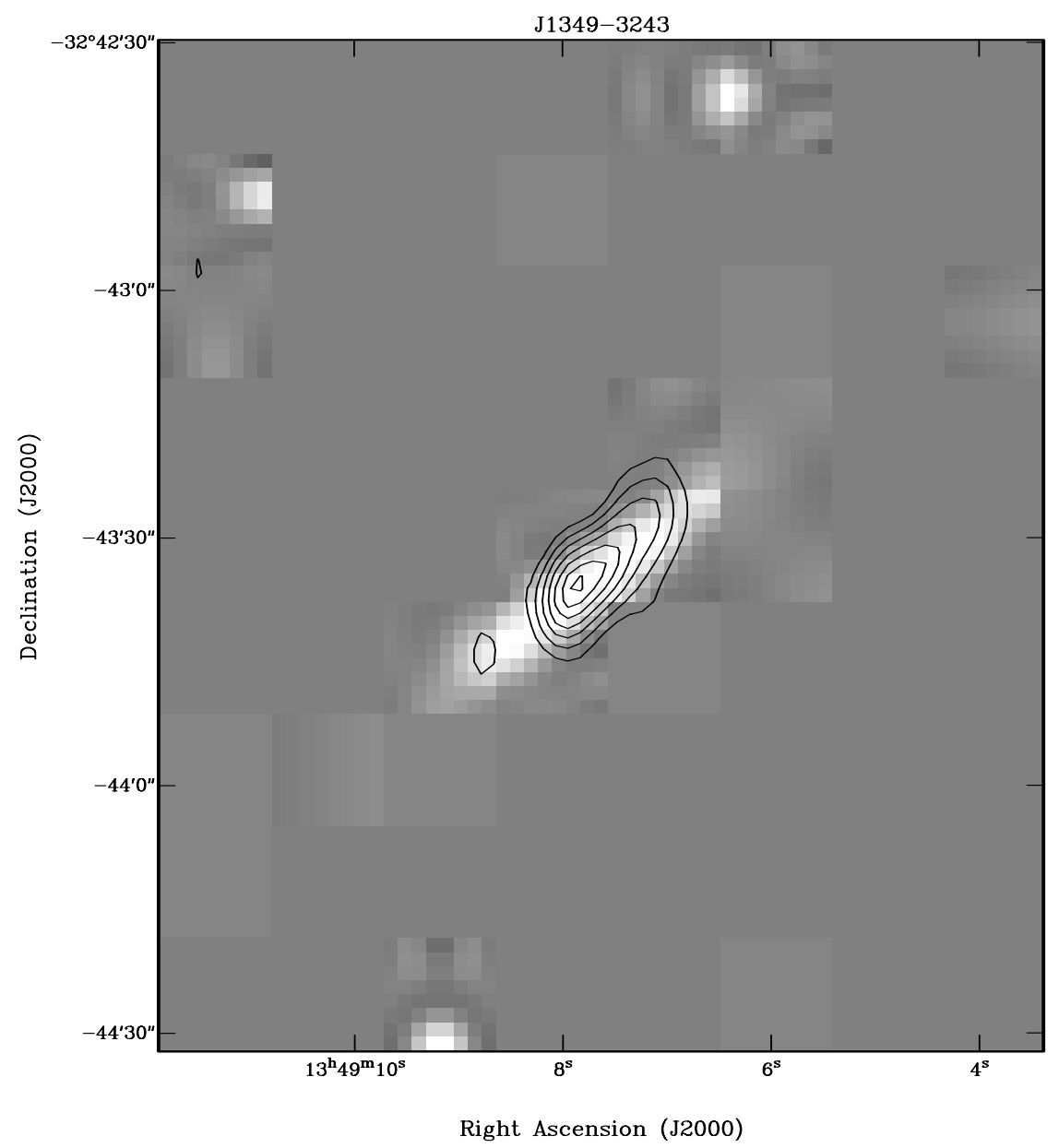

Fig. 6. $22 \mathrm{~cm}$ radio image of J1349-3243 overlaid on the DSS optical frame. The contours are $-0.32,0.32,0.48,0.64,0.80$, $0.96,1.10 \mathrm{mJy}$. The restoring beam is $11.24^{\prime \prime} \times 5.27^{\prime \prime}$, in $\mathrm{PA}-7^{\circ}$.

statistically negligible. This result is visible also from Fig. 4, which shows that the radio sources are fairly uniformly distributed over the whole complex, regardless of the underlying optical density.

\section{Discussion and conclusions}

This work is part of a larger project whose aim is to study the effects of cluster mergers at radio wavelengths in the central region of the Shapley Concentration. The results obtained for the two major merging complexes have already been presented and discussed (Venturi et al. 2000, 2001). Here we report on ATCA $22 \mathrm{~cm}$ observations of the third merging complex in the Shapley Concentration, formed by the three clusters A3571, A3572 and A3575. In particular in this paper we have focussed on the possible influence of cluster mergers on the statistical properties of the radio galaxies. We can briefly summarise our results as follows.

(a) Six radio galaxies from our $22 \mathrm{~cm}$ radio sample were identified with galaxies in the in the A3571 chain. Other nine, associated with galaxies brighter than $b_{j} \leq 18.5$ and with no measured redshift, are possible Shapley member candidates. (b) All Shapley radio galaxies and candidates are weak radio sources, with radio powers in the range $21.2<\log P_{22}$ $\left(\mathrm{W} \mathrm{Hz}^{-1}\right)<22.6$. They are located in A3571 and in A3575 (none in A3572).

(c) The estimate of the RLF based on the analysis by LO96 points towards a lack of radio galaxies in the A3571 complex when we normalise the number of radio galaxies to the whole chain. However, if we take into account that all the radio galaxies considered in the RLF are located within $0.5 R_{\mathrm{A}}$ from the centre of A3571, the number of detections is in very good agreement with the expectations.

(d) The radio source counts in this region are dominated by the background counts, despite an optical overdensity of $\sim 1.5$.

Our results suggest a dual character of the A3571 cluster complex, where the A3571 cluster alone shows different properties than the chain as a whole, as clear from points (b) and (c) above. The same dual properties are evident also from observations at other wavelengths.

The cluster gas in A3571 is very hot $(T=7.6 \mathrm{keV})$ and luminous $\left(L_{\mathrm{X}, \mathrm{bol}} \sim 4 \times 10^{44} \mathrm{erg} \mathrm{s}^{-1}\right.$, Ettori et al. 1997). With the observed temperature we can estimate the expected galaxy velocity dispersion, according to the 


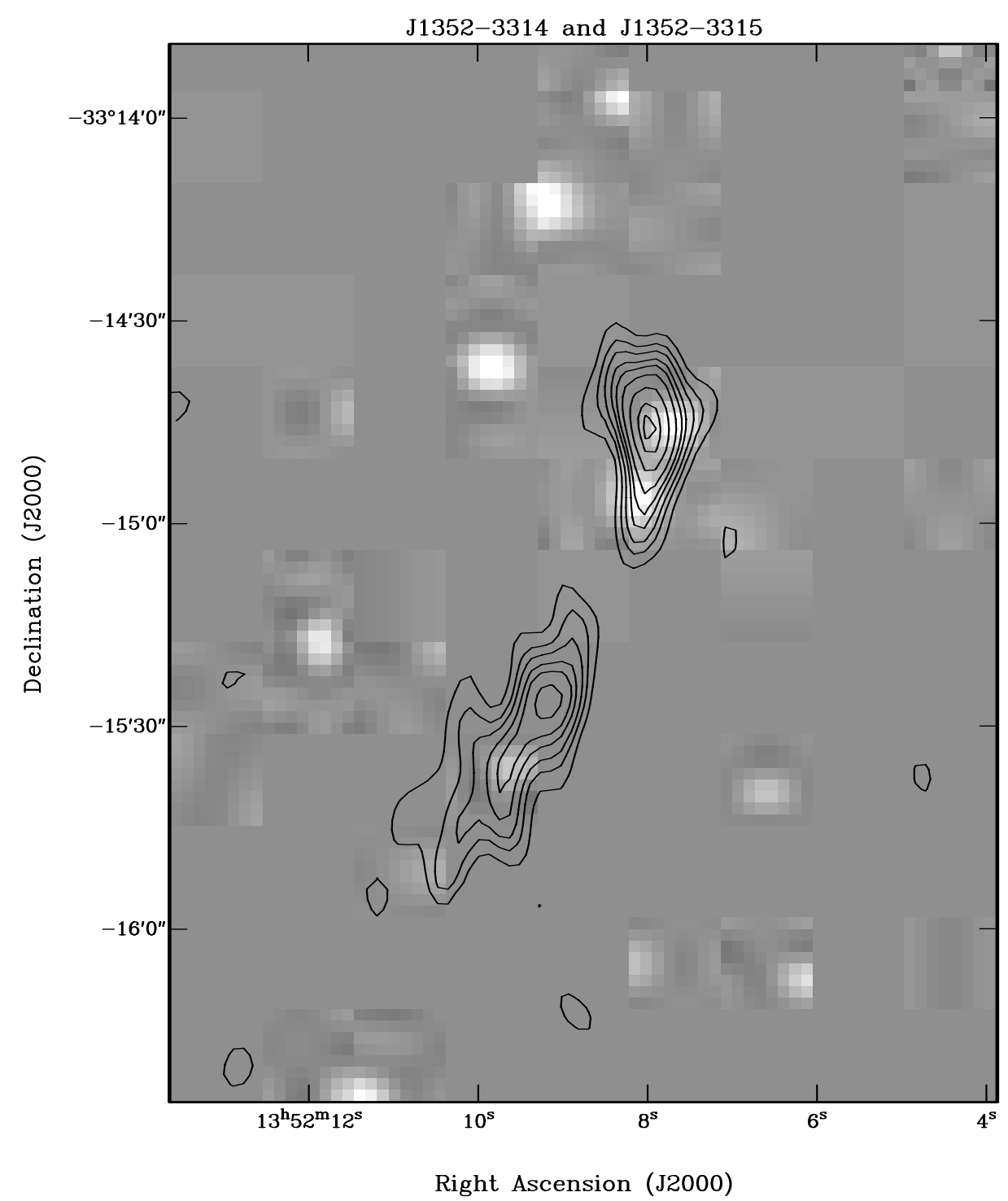

Fig. 7. $22 \mathrm{~cm}$ radio image of J1352-3314 and J1352-3315 overlaid on the DSS optical frame. The contours are $-1.10,1.10$, $1.70,2.20,2.80,3.30,4.00,5.00,6.00,6.50 \mathrm{mJy}$. The restoring beam is $11.24^{\prime \prime} \times 5.27^{\prime \prime}$, in $\mathrm{PA}-7^{\circ}$.

following formula (Lubin \& Bahcall 1993):

$\sigma_{v}=10^{2.52 \pm 0.07}(k T)^{0.6 \pm 0.11}$

which gives a value $\sigma_{v} \sim 1118 \mathrm{~km} \mathrm{~s}^{-1}$. This is in good agreement with $\sigma_{v} \sim 1022 \mathrm{~km} \mathrm{~s}^{-1}$, obtained by Quintana \& De Souza (1993) on the basis of redshift measurements, and suggests that A3571 is relaxed, as proposed by Nevalainen et al. (2000). Another piece of evidence in favour of virialisation is the presence of a cooling flow in its innermost region (Peres et al. 2000), which requires that the cluster has been in equilibrium at least since $4^{-}$ $6 \times 10^{9}$ yrs.

Conversely, the redshift survey of Quintana et al. (1997), together with the presence of the giant cD galaxy at the centre of A3571 (MGC05-33-002) suggest that the whole chain is not relaxed and that a recent merger may be responsible for the formation of the central $\mathrm{cD}$ galaxy.

Inspection of the optical isodensity contours given in Fig. 1 shows a major optical overdensity in A3571, while the other two clusters are not as well defined (especially A3575). In the light of the optical distribution and of the results summarised above, it is very important to understand whether A3575 and A3572 are the debris of a merger event, or if they are smaller entities yet to interact with the massive A3571.

The results obtained from our $22 \mathrm{~cm}$ survey may help in clarifying the situation.

It has been argued that a connection exists between cluster mergers and a high number of starburst radio galaxies. In particular Owen et al. (1999), in a comparative study of the two clusters A2125 and A2645, suggested that merging could be responsible both for the high fraction of blue galaxies and of radio AGN in A2125, compared to the relaxed A2645.

The results presented in this paper are not conclusive in this respect, mainly because of the lack of complete spectroscopy in this region of the sky, however there 


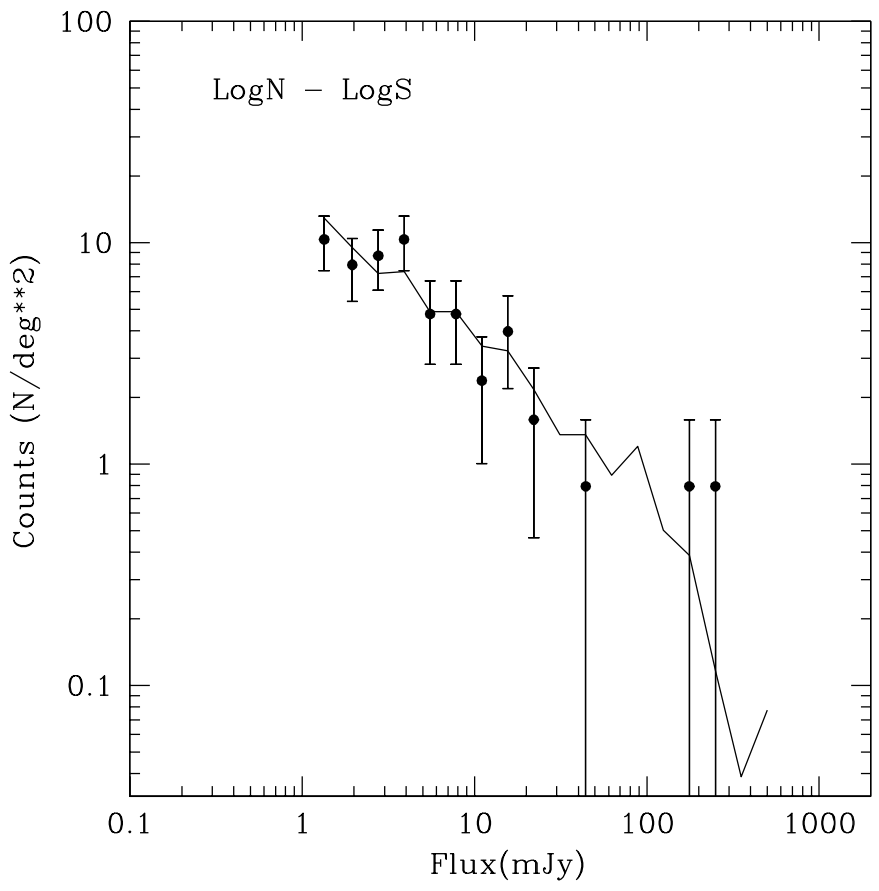

Fig. 8. $\log N-\log S$. Dots refer to counts in the A3571 complex; the solid line represents the background distribution (Prandoni et al. 2001).

is indication that a considerable fraction of candidate starburst galaxies is located in A3571. In particular, we found 9 galaxies brighter than $b_{j} \leq 18.5$ (including confirmed members and candidates) characterised by low radio power, i.e. $\log P_{22}\left(\mathrm{~W} \mathrm{~Hz}^{-1}\right) \leq 21.78$, whose radio emission could be driven by star formation. These numbers should be compared to the 12-13 starburst galaxies detected in the richness 4 distant cluster A2125 by Owen et al. (1999). It is crucial that redshift and spectral information becomes available for the objects in the A3571 complex, to confirm that we are indeed observing a high fraction of starburst galaxies. We note that this result is considerably different from what we obtained for the A3528 and A3558 complexes, where no hint of such excess was revealed (Venturi et al. 2000, 2001) at similar radio power limits.

We propose that A3571 is the result of a recent merger event, at the very last stage of its evolution, and that the three main cluster complexes in the central region of the Shapley Concentration are part of an evolutionary sequence. In particular, the wealth of information available from radio to optical (both photometry and spectroscopy), up to $\mathrm{X}$-ray energies, suggest the following scenario:

(i) The A3528 cluster complex is at the very beginning of a merger event, where the two merging entities have just started "to feel each other". The gradients in the temperature distribution of the intracluster gas delineate the merging front (Schindler 1996). The radio luminosity function of elliptical galaxies is in good agreement with that of ellipticals not located in merging clusters (LO96), and no sign of starburst emission, possibly induced by merger shocks, is detected (Venturi et al. 2001). We suggested that the pre-merging stage had not yet had time to affect the radio emission properties of the cluster galaxies in the complex.

(ii) The A3558 complex is thought to be an advanced merger, where two clusters of similar mass have already undergone the first core-core encounter. The amazing similarity between the galaxy distribution (Bardelli et al. 1998) and the gas density distribution (Ettori et al. 1997; Kull \& Böhringer 1999) provides further evidence of the ongoing process. In the radio band it was found (Venturi et al. 2000) that this complex shows a significant deficit of radio galaxies in comparison with the radio luminosity function of LO96, suggesting that the major encounter switched off the nuclear radio emission and temporarily inhibited its formation. No radio excess of starburst origin was detected in the shock region, however data from a deeper survey in the same region are being analysed (Venturi et al. in prep.).

(iii) We suggest that the A3571 complex represents the final stage of a merger event, where A3571 itself is the resulting cluster after virialization of the merger. The distribution of the gas is already relaxed, as well as the galaxy distribution in A3571, while the outer edge of the galaxy distribution is still unrelaxed. The radio properties reflect the different dynamical stage of the central relaxed region of the complex (the cluster A3571) and the active dynamics of the outskirts. The location of the radio galaxies in A3571 suggests that they had time to develop a nuclear source after the active merging ceased.

We note that the distribution of the optical galaxies and of the $\mathrm{X}$-ray emitting gas in these three cluster complexes is remarkably similar to the various stages of the clustercluster collision recently modelled by Ricker \& Sarazin (2001). Their simulations for a frontal merger of two clusters with mass ratio $M_{1} / M_{2}=1$ show an A3528-like situation for $t=0$, which evolves into an A3558 scenario at $t \sim 5$ Gyr, to end up in the A3571 case after $\sim 9$ Gyr.

Acknowledgements. SB acknowledges support from the ASI grant ASI-I/R/037/01. The authors wish to thank R. Stathakis for his help in the compilation of the optical catalogue. This work has made use of the NASA Extragalactic Database NED. The Australia Telescope Compact Array is operated by the Australia Telescope National Facility.

\section{References}

Abell, G. O., Corwin, H. G., \& Olowin, R. P. 1989, A\&AS, 70, 1 (ACO)

Baldi, A., Bardelli, S., \& Zucca, E. 2001, MNRAS, 324, 509

Bardelli, S., Zucca, E., Malizia, A., et al. 1996, A\&A, 305, 435

Bardelli, S., Zucca, E., Zamorani, G., Vettolani, G., \& Scaramella, R. 1998, MNRAS, 296, 599

Brunetti, G., Setti, G., Feretti, L., \& Giovannini, G. 2001, MNRAS, 320, 365

Buote, D. A. 2001, ApJ, 533, L15

Condon, J. J., Cotton, W. D., Greisen, E. W., \& Yin, F. 1998, AJ, 115, 1693 
Drinkwater, M. J., Proust, D., Parker, Q. A., Quintana, H., \& Slezak, E. 1999, PASA, 16, 113

Dwarakanath, K. S., \& Owen, F. N. 1999, AJ, 118, 625

Edge, A. C., Stewart, G. C., Fabian, A. C., \& Arnaud, K. A. 1990, MNRAS, 245, 559

Ensslin, T. A., \& Brüggen, M. 2002, MNRAS, in press

Ettori, S., Fabian, A. C., \& White, D. A. 1997, MNRAS, 289, 787

Fanaroff, B. L., \& Riley, J. M. 1974, MNRAS, 167, 31

Feretti, L., \& Giovannini, G. 1996, in Extragalactic Radio Sources, ed. R. Ekers, C. Fanti, \& L. Padrielli (Kluwer Academic Publ.), IAU Symp., 175, 133

Feretti, L., \& Giovannini, G. 2002, in Cluster Mergers, ed. L. Feretti, I. M. Gioia, \& G. Giovannini (Kluwer Academic Publ.), in press

Girardi, M., Giuricin, G., Mardirossian, F., Mezzetti, M., \& Boschin, W. 1998, ApJ, 505, 74

Hanami, H., Tsuru, T., Shimasaku, K., et al. 1999, ApJ, 521, 90

Kemp, S. N., \& Meaburn, J. 1991, MNRAS, 251, 10

Kull, A., \& Böhringer, H. 1999, A\&A, 341, 23

Ledlow, M. J., \& Owen, F. N. 1996, ApJ, 112, 9 (LO96)

Lubin, L. M., \& Bahcall, N. A. 1993, ApJ, 415, 17

Maddox, S. J., Efstathiou, G., Sutherland, W. J., \& Loveday, J. 1990, MNRAS, 243, 692

Metcalfe, N., Godwin, J. G., \& Peach, J. V. 1994, MNRAS, 267,431

Narayan, R., \& Yi, I. 1995, ApJ, 452, 710

Nevalainen, J., Markevitch, M., \& Forman, W. 2000, ApJ, 536, 73

Nevalainen, J., Kaastra, J., Parmar, A. N., et al. 2001, A\&A, 369,459

Owen, F. N., Ledlow, M. J., Keel, W. C., \& Morrison, G. E. 1999, AJ, 118, 633
Peres, C. B., Fabian, A. C., Edge, A. C., et al. 1998, MNRAS, 298, 416

Prandoni, I., Gregorini, L., Parma, P., et al. 2000a, A\&A, Suppl. Ser., 146, 31

Prandoni, I., Gregorini, L., Parma, P., et al. 2000b, A\&A, Suppl. Ser., 146, 41

Prandoni, I., Gregorini, L., Parma, P., et al. 2001, A\&A, 365, 392

Quintana, H., \& de Souza, R. 1993, A\&AS, 101, 475

Quintana, H., Melnick, J., Proust, D., \& Infante, L. 1997, A\&AS, 125, 247

Ricker, P. M., \& Sarazin, C. L. 2001, ApJ, 561, 621

Sarazin, C. L. 2000, in Constructing the Universe with Clusters of Galaxies, ed. F. Durrett, \& D. Gerbal, electronic proc. http://www.iap.fr/Conferences/Colloque/coll2000/ contributions

Sault, R. J., Teuben, P. J., \& Wright, M. C. H. 1995, in Astronomical Data Analysis Software and Systems. IV, ed. R. Shaw, H. E. Peyne, \& J. J. E. Hayes, ASP Conf. Ser., 77, 433

Schindler, S. 1996, MNRAS, 280, 309

Unewisse, A. M., Hunstead, D. W., \& Pietrzynski, B. 1993, Pubbl. Astron. Soc. Austr., 10, 229

Venturi, T., Bardelli, S., Morganti, R., \& Hunstead, R. W. 2000, MNRAS, 314, 594

Venturi, T., Bardelli, S., Zambelli, G., Morganti, R., \& Hunstead, R. W. 2001, MNRAS, 324, 1131

Yentis, D. J., Cruddace, R. G., Gursky, H., et al. 1992, in Digitised Optical Sky Surveys, ed. H. T. MacGillivray, \& E. B. Thomson (Kluwer Academic Publishers), 67

Zucca, E., Zamorani, G., Scaramella, R., \& Vettolani, G. 1993, ApJ, 407, 470 